Pseudo completions and completions in stages of o-minimal structures

Tressl, Marcus

2006

MIMS EPrint: 2007.187

Manchester Institute for Mathematical Sciences

School of Mathematics

The University of Manchester

\footnotetext{
Reports available from: http://eprints.maths.manchester.ac.uk/

And by contacting: The MIMS Secretary

School of Mathematics

The University of Manchester

Manchester, M13 9PL, UK
} 


\title{
Pseudo completions and completions in stages of o-minimal structures
}

\author{
Marcus Tressl
}

Received: 26 June 2004 / Published online: 14 July 2006

(C) Springer-Verlag 2006

\begin{abstract}
For an o-minimal expansion $R$ of a real closed field and a set $\mathscr{V}$ of $T h(R)$-convex valuation rings, we construct a "pseudo completion" with respect to $\mathscr{V}$. This is an elementary extension $S$ of $R$ generated by all completions of all the residue fields of the $V \in \mathscr{V}$, when these completions are embedded into a big elementary extension of $R$. It is shown that $S$ does not depend on the various embeddings up to an $R$-isomorphism. For polynomially bounded $R$ we can iterate the construction of the pseudo completion in order to get a "completion in stages" $S$ of $R$ with respect to $\mathscr{V} . S$ is the "smallest" extension of $R$ such that all residue fields of the unique extensions of all $V \in \mathscr{V}$ to $S$ are complete.
\end{abstract}

Mathematics Subject Classification (2000) Primary: 03C64 · 12J10 · 12J15; Secondary: 13B35

Let $R$ be a real closed field. There is a largest ordered field $\hat{R}$ such that $R$ is dense in $\hat{R} . \hat{R}$ is again real closed and $\hat{R}$ is called the completion of $R$ (cf. [7]). If $v$ is a proper real valuation on $R$, then $\hat{R}$ is also the underlying field of the completion of the valued field $(R, v)$ and $\hat{R}$ is obtained by adjoining limits of Cauchy sequences with respect to $v$ as explained in [8].

We generalize this construction as follows. Let $\mathscr{V}$ be a set of convex valuation rings, possibly containing $R$ itself. We construct a "smallest" real closed

Partially supported by the European RTNetwork RAAG (contract no. HPRN-CT-2001-00271).

M. Tressl ( $\otimes)$

NWF-I Mathematik, Universität Regensburg, 93040 Regensburg, Germany

e-mail: marcus.tressl@mathematik.uni-regensburg.de 
field containing $R$ which has a limit for all sequences of $R$ that become Cauchy sequences after passing to the residue field of some $V \in \mathscr{V}$. This can also be done for o-minimal expansions of real closed fields and $T h(R)$-convex valuation rings (see Sect. 3 for the definition of the completion in this case).

Our first result (Theorem 4.1) basically says that we can adjoin the missing limits to $R$ in any order and that the resulting elementary extension $R^{\prime}$ of $R$ does not depend on the choices, up to an $R$-isomorphism. We call $R^{\prime}$ the pseudo completion of $R$ with respect to $\mathscr{V}$. If $R$ is a pure real closed field (more generally, a polynomially bounded o-minimal expansion of a real closed field), then we can compute the value groups and the residue fields of convex valuation rings of $R^{\prime}$. Moreover for every valuation ring $V \in \mathscr{V}$ the convex hull $V^{\prime}$ of $V$ in $R^{\prime}$ is the unique convex valuation ring of $R^{\prime}$, lying over $V$.

It turns out that $R^{\prime}$ is not "complete in stages" with respect to $\mathscr{V}^{\prime}:=\left\{V^{\prime} \mid V \in\right.$ $\mathscr{V}\}$ in general, i.e. not all residue fields of the $V^{\prime}$ are complete in general [cf. Example 5.7]. Therefore, in order to get a "smallest" extension of $R$, which is complete in stages, we have to iterate the construction of the pseudo completion. The iteration stops at an ordinal and the resulting extension $S$ of $R$ is called the completion in stages of $R$ with respect to $\mathscr{V}$. In Theorem 5.10, we compute the value groups and the residue fields of convex valuation rings of $S$. Moreover in Theorem 5.10 it is shown that every element $s \in S \backslash R$ is of the form $a x+b$ where $a, b \in R$ and $x \in S$ such that for a unique convex valuation ring $W$ of $S$ with $W \cap R \in \mathscr{V}, s / \mathfrak{m}_{W}$ is the limit of a Cauchy sequence of $V / \mathfrak{m}_{V}$ without limits in $V / \mathfrak{m}_{V}$; here $\mathfrak{m}_{V}, \mathfrak{m}_{W}$ denote the maximal ideal of $V, W$, respectively.

Finally we want to point out a combinatorial tool which we use in our arguments. This is a dimension in o-minimal structures, we call it the realization rank, which is coarser than the ordinary dimension associated to o-minimal structures. For real closed fields $R \subseteq S$, with tr.deg. $S / R$ finite, the realization rank of $S$ over $R$ is the maximal number of elements $s_{1}, \ldots, s_{k} \in S$ such that $\operatorname{tp}\left(s_{1}, \ldots, s_{k} / R\right)$ is uniquely determined by the open boxes contained in it [cf. Proposition 1.15]. We first analyze this new dimension.

The explanation of the valuation theoretic notions and facts used for o-minimal expansions of fields can be found in [2]. Readers who are mainly interested in the case of real closed fields may replace "o-minimal structure" by "real closed field", "definable" by "semi-algebraic" and "definable closure" by "real closure". Moreover if $R \subseteq S$ are real closed fields and $B \subseteq S$, then the type $\operatorname{tp}(B / R)$ of $B$ over $R$ can be identified with the ordering of $R\left[t_{b} \mid b \in B\right]$ (where the $t_{b}$ are indeterminates) induced by the evaluation map $t_{b} \mapsto b$.

\section{The realization rank}

We start with a reminder on dependence relations as in van der Waerden's "Algebra" ([10]).

Definition 1.1 A relation $x \ll A$ between elements $x$ and subsets $A$ of a given set $X$ is called $a$ dependence relation if the following conditions are fulfilled:

(D1) $x \ll\{x\}$. 
(D2) if $x \ll A$ and $A \subseteq B$ then $x \ll B$.

(D3) if $x \ll A$ then there is a finite subset $B$ of $A$, such that $x \ll B$.

(D4) (exchange lemma) if $A$ is finite, $x \ll A \cup\{y\}$ and $x \ll A$, then $y \ll A \cup\{x\}$.

(D5) (transitivity) if $A$ is finite, $x \ll A$ and $a \ll B$ for every $a \in A$, then $x \ll B$.

We rephrase this notion in terms of independent sets:

Definition 1.2 Let $X$ be a set and let $\mathcal{I}$ be a nonempty set of finite subsets of $X$. $\mathcal{I}$ is called $a$ system of independence if the following two properties hold.

(I1) If $A \subseteq B \in \mathcal{I}$ and $B \in \mathcal{I}$, then $A \in \mathcal{I}$.

(I2) If $A, B \in \mathcal{I}, x \in X \backslash B$ and if $B \cup\{x\} \in \mathcal{I}$, then $A \cup\{x\} \in \mathcal{I}$ or there is some $a \in A \backslash B$ such that $B \cup\{a\} \in \mathcal{I}$.

Observe that $\emptyset \in \mathcal{I}$ if $\mathcal{I}$ is an independence system. Dependence relations and systems of independence describe the same concept:

Proposition 1.3 If $\mathcal{I}$ is a system of independence of a set $X$ then we define a relation between elements and subsets of $X$ by

$x \ll_{\mathcal{I}} A: \Longleftrightarrow x \in A$ or there is some $A_{0} \subseteq A, A_{0} \in \mathcal{I}$ such that $A_{0} \cup\{x\} \notin \mathcal{I}$.

If $\ll$ is a dependence relation of $X$ then we define

$$
\mathcal{I}(\ll):=\{A \mid A \text { is finite and } a \nless A \backslash\{a\} \text { for all } a \in A\} \text {. }
$$

(i) If $\ll$ is a dependence relation of $X$, then $\mathcal{I}(\ll)$ is a system of independence and

$$
\ll \mathcal{I}(\ll)=\ll \text {. }
$$

(ii) If $\mathcal{I}$ is a system of independence of $X$, then $\ll_{\mathcal{I}}$ is a dependence relation and

$$
\mathcal{I}\left(\ll_{\mathcal{I}}\right)=\mathcal{I} \text {. }
$$

Proof This is a folklore fact, we omit the easy proof.

If $\mathcal{I}$ is a system of independence of $X$ with corresponding dependence relation $\ll$ and $A \subseteq X$, then we write $\mathcal{I}-\operatorname{rk}(A)$ or $\ll-\operatorname{rk}(A)$ respectively, for the cardinality of a basis - i.e. a maximal $\ll$-independent subset-of $A$.

\subsection{The realization rank}

We always work with small subsets of a large $o$-minimal structure $\mathfrak{M}$ expanding a dense linear order without endpoints; that means $\mathfrak{M}$ will be $\lambda$-big for some 
large infinite cardinal $\lambda$, whereas "small" means "of cardinality $\lambda$ " (cf. [4], 10.1). $\mathfrak{M}$ is not mentioned always.

Moreover we fix a (small) subset $A$ of $\mathfrak{M}$. $A$ is always assumed to be definably closed. For a set $X, \operatorname{cl}(X)$ denotes the definable closure of $X$ (in $\mathfrak{M})$. If $D \subseteq \mathfrak{M}$ is definably closed, then $D\langle X\rangle$ also denotes $\operatorname{cl}(D \cup X)$.

Lemma 1.4 If $p$ is a 1-type over $A$ and $A \subseteq B \subseteq \mathfrak{M}$, then the following conditions are equivalent.

(i) $p$ has a unique extension to $B$.

(ii) If $p$ is realized in $\mathrm{cl}(B)$ then $p$ is realized in $A$.

Proof The set $A$ is definably closed. Therefore each formula with parameters in $A$ with one free variable is equivalent to a quantifier free formula of the language $\{<\}$ with parameters in $A$. Now the lemma follows easily.

Definition 1.5 If $B$ is a subset of $\mathfrak{M}$ and if $c$ is an element from $\mathfrak{M}$, we say that $c$ is dominated by $B$ over $A$ (or $A$-dominated by $B$ ) and write $c \triangleleft_{A} B$, if $\operatorname{tp}(c / A)$ is realized in $\mathrm{cl} A \cup B$; otherwise $c$ is called $A$ - indominated by $B$.

Counterexample 1.6 A-dominance is not a dependence relation, since transitivity is violated. To see an example let $\mathfrak{M}$ be a big real closed field containing $\mathbb{R}$, take $A=R_{0}$ to be the real closure of $\mathbb{Q}$ and let $\mu \in \mathfrak{M}$ be positive and infinitesimal over $\mathbb{R}$. Then

(a) $\mu \in R_{0}(\pi, \pi+\mu)$, thus $\mu$ is $R_{0}$-dominated by $\{\pi, \pi+\mu\}$.

(b) $\pi+\mu$ is $R_{0}$-dominated by $\{\pi\}$.

(c) $\mu$ is $R_{0}$-indominated by $\{\pi\}$.

In spite of this example, the $A$-dominance relation leads to a dependence relation. Before introducing this relation we prove that $\triangleleft_{A}$ satisfies axioms (D1)(D4) of a dependence relation. We suppress the index $A$ and write dominated or indominated only. The set $A$ is always fixed and, as mentioned in the beginning, definably closed.

Certainly we have for all $c \in \mathfrak{M}$ and all $B, C \subseteq \mathfrak{M}$ :

(D1) $c$ is dominated by $\{c\}$.

(D2) $c$ dominated by $B, B \subseteq C \Rightarrow c$ dominated by $C$.

(D3) if $c$ is dominated by $B$, then there is a finite subset $B_{0}$ of $B$, such that $c$ is dominated by $B_{0}$.

From Lemma 1.4 we know for any element $c \notin A$ the equivalence of

(i) $c$ is indominated by $B$.

(ii) $\operatorname{tp}(c / A \cup B)$ is the unique extension of $\operatorname{tp}(c / A)$ on $A \cup B$.

(iii) If $c^{\prime} \in \mathfrak{M}$ such that $\operatorname{tp}(c / A)=\operatorname{tp}\left(c^{\prime} / A\right)$, then $c^{\prime} \notin \operatorname{cl} A \cup B$.

Exchange Lemma for $A$-dominance 1.7 If $c$ is indominated by $B$ and dominated by $B d$, then $d$ is dominated by $B c$. 
Proof We search for a realization of $\operatorname{tp}(d / A)$ in $\operatorname{cl}(A B c)$. Since $c$ is dominated by $B d$ there is some realization $c^{\prime} \in \operatorname{cl}(A B d)$ of $t p(c / A)$. Since $c$ is indominated by $B$, it follows that $c^{\prime} \notin \operatorname{cl}(A B)$. From the exchange lemma for the definable closure "cl" in o-minimal structures (cf. [6], Theorem 4.1) we get $d \in \operatorname{cl}\left(A B c^{\prime}\right)$. Since $c$ is indominated by $B$ and $t\left(c^{\prime} / A\right)=t(c / A)$ it follows from the equivalence preceding our lemma that $t p(c / A B)=\operatorname{tp}\left(c^{\prime} / A B\right)$. Let $\sigma$ be an $(A \cup B)$-automorphism of $\mathfrak{M}$ such that $\sigma\left(c^{\prime}\right)=c$. Then $\sigma(d) \in \operatorname{cl} A B c$ is a realization of $\operatorname{tp}(d / A)$ as desired.

The next proposition implies a variant of transitivity for $A$-dominance, which we will use to define a system of independence.

Proposition 1.8 Let I be an index set, let $\left\{b_{i} \mid i \in I\right\}, C$ and $D$ be sets such that for each $i \in I, b_{i}$ is indominated by $C \cup\left\{b_{j} \mid j \neq i\right\}$. Suppose $b_{i}$ is dominated by $C \cup D$ for every $i \in I$. Then $\operatorname{tp}\left(\left(b_{i}\right)_{i \in I} / A \cup C\right)$ is realized in $\mathrm{cl} A \cup C \cup D$. More precisely: If $b_{i}^{\prime}$ is a realization of $\operatorname{tp}\left(b_{i} / A\right)$ in $\mathrm{cl} A \cup C \cup D$, then $\left(b_{i}^{\prime}\right)_{i \in I}$ is a realization of $\operatorname{tp}\left(\left(b_{i}\right)_{i \in I} / A \cup C\right)$.

Proof We have $b_{i} \neq b_{j}$ if $i \neq j$ and it is enough to prove the Proposition for finite $I$. We do an induction on $n=\operatorname{card} I$ :

$n=1$ : suppose $b$ is dominated by $C \cup D$, indominated by $C$ and $b^{\prime}$ realizes $\operatorname{tp}(b / A)$ in cl $A \cup C \cup D$. Since $b$ is indominated by $C$ the type $t p(b / A \cup C)$ is realized by $b^{\prime}$ too.

$n \rightarrow n+1$. Suppose $\left\{b_{1}, \ldots, b_{n+1}\right\}$ is indominated by $C$ and $b_{i}$ is dominated by $C \cup D$. Let $b_{1}^{\prime}, \ldots, b_{n+1}^{\prime} \in \mathrm{cl} A \cup C \cup D$ be realizations of $t p\left(b_{1} / A\right), \ldots, t p\left(b_{n+1} / A\right)$ respectively. By the induction hypothesis we have $t p\left(b_{1}, \ldots, b_{n} / A \cup C\right)=$ $\operatorname{tp}\left(b_{1}^{\prime}, \ldots, b_{n}^{\prime} / A \cup C\right)$.

Let $\sigma$ be an $A \cup C$-automorphism of $\mathfrak{M}$ such that $\sigma\left(b_{i}\right)=b_{i}^{\prime}(1 \leq i \leq n)$. Since $b_{n+1}$ is indominated by $C \cup\left\{b_{1}, \ldots, b_{n}\right\}$, we see that $\sigma\left(b_{n+1}\right)$ is indominated by $C \cup\left\{b_{1}^{\prime}, \ldots, b_{n}^{\prime}\right\}$, that is $\operatorname{tp}\left(f\left(b_{n+1}\right) / A \cup C \cup\left\{b_{1}^{\prime}, \ldots, b_{n}^{\prime}\right\}\right)=\operatorname{tp}\left(b_{n+1}^{\prime} / A \cup C \cup\right.$ $\left.\left\{b_{1}^{\prime}, \ldots, b_{n}^{\prime}\right\}\right)$. Hence $\left(b_{1}^{\prime}, \ldots, b_{n+1}^{\prime}\right)$ is a realization of $t p\left(b_{1}, \ldots, b_{n+1} / A \cup C\right)$.

Corollary and Definition 1.9 Let $A, C \subseteq \mathfrak{M}$ and let $A$ be definably closed. For elements $x \in \mathfrak{M}$ and subsets $B$ of $\mathfrak{M}$ we define $x \triangleleft_{A, C} B: \Longleftrightarrow x \triangleleft_{A}(C \cup B)$. Then $\triangleleft_{A, C}$ satisfies properties (D1)-(D4) of a dependence relation (cf. Definition 1.1).

Proof Properties (D1)-(D3) are obviously true for $\triangleleft_{A, C}$. (D4) holds by Exchange Lemma for $A$-dominance 1.7.

Definition 1.10 Let $A, C \subseteq \mathfrak{M}$ and let $A$ be definably closed. We define

$$
\mathcal{I}(A, C):=\left\{B \subseteq \mathfrak{M} \mid B \text { is finite and for all } b \in B \text { we have } b \oiint_{A, C} B \backslash\{b\}\right\} \text {. }
$$

Proposition 1.11 $\mathcal{I}(A, C)$ is a system of independence.

Proof Certainly, property (I1) of an independence system holds for $\mathcal{I}(A, C)$ and we show that also property (I2) of an independence system holds for $\mathcal{I}(A, C)$. 
To see this let $B, D \in \mathcal{I}(A, C)$ and let $x \notin D$. Suppose $B \cup\{x\} \notin \mathcal{I}(A, C)$ and $D \cup\{b\} \notin \mathcal{I}(A, C)$ for all $b \in B$. We have to show $D \cup\{x\} \notin \mathcal{I}(A, C)$. Since $\triangleleft_{A, C}$ satisfies (D1)-(D4) (cf. Corollary and Definition 1.9), this means $x \triangleleft_{A, C} B$ and $b \triangleleft_{A, C} D$ for all $b \in B$. As $B \in \mathcal{I}(A, C)$ we can apply Proposition 1.8:

Let $B=\left\{b_{1}, \ldots, b_{n}\right\}$ and let $F$ be an $A \cup C$-definable map, such that $F\left(b_{1}, \ldots, b_{n}\right)$ is a realization of $\operatorname{tp}(x / A)$. From Proposition 1.8 we know that $\operatorname{tp}\left(b_{1}, \ldots, b_{n} / A \cup C\right)$ is realized in $\operatorname{cl} A \cup C \cup D$ by some $n$-tuple $\left(b_{1}^{\prime}, \ldots, b_{n}^{\prime}\right)$. If $\sigma$ is an $A \cup C$-automorphism of $\mathfrak{M}$ such that $\sigma\left(b_{i}\right)=b_{i}^{\prime}$ then $\sigma\left(F\left(b_{1}, \ldots, b_{n}\right)\right)=$ $F\left(b_{1}^{\prime}, \ldots, b_{n}^{\prime}\right)$ is a realization of $\operatorname{tp}(x / A)$ in $\operatorname{cl} A \cup C \cup D$.

Hence $x \triangleleft_{A, C} D$ and $D \cup\{x\} \notin \mathcal{I}(A, C)$ as desired.

Notations 1.12 The dependence relation corresponding to $\mathcal{I}(A, C)$ as explained in Proposition 1.3 is denoted by $\ll_{A, C}$. The dimension associated with $\ll_{A, C}$ is denoted by $\mathrm{rk}_{A, C}$ and is called the realization rank with respect to $A, C$.

If the set $C$ is contained in $A$ we write $\ll_{A}$ and $\mathrm{rk}_{A}$ instead of $\ll_{A, C}$ and $\mathrm{rk}_{A, C}$. $A$ set $B$ is called $\ll_{A, C}$-independent if every finite subset of $B$ is in $\mathcal{I}(A, C)$.

Proposition 1.13 We have for every set $B \subseteq \mathfrak{M}$ :

(i) $B$ is $\ll_{A, C}$-independent if and only if $b \oiint_{A, C} B \backslash\{b\}$ for all $b \in B$.

(ii) For all $x \in \mathfrak{M}, x \ll_{A, C} B \Longleftrightarrow x \triangleleft_{A, C} B_{0}$ for some $\ll_{A, C}$-independent subset $B_{0}$ of $B$.

(iii) $\operatorname{rk}_{A, C}(B)=\min \left\{\right.$ card $B_{0} \mid B_{0} \subseteq B$ and $b \triangleleft_{A, C} B_{0}$ for all $\left.b \in B\right\}$

Proof (i) holds by definition of $\ll_{A, C}$ and since $\triangleleft_{A, C}$ satisfies (D1)-(D4) and (ii) is implied by (i).

(iii) $\geq$ holds, since by (ii), for a $\ll_{A, C}$-basis $B_{0}$ of $B$ we have $b \triangleleft_{A, C} B_{0}$ for all $b \in B$.

Conversely let $B^{\prime}$ be a $\ll_{A, C}$-basis of $B$ and let $B_{0} \subseteq B$, such that each $b \in B$ is $A$-dominated by $B_{0} \cup C$. By Proposition 1.8 the type of $B^{\prime}$ over $A \cup C$ is realized in $\operatorname{cl}\left(A \cup C \cup B_{0}\right)$. Since $\operatorname{dim} B^{\prime} / A \cup C=$ card $B^{\prime}$ it follows that $\operatorname{dim} B_{0} / A \cup C \geq \operatorname{card} B^{\prime}$. Hence $\operatorname{rk}_{A, C} B=\operatorname{card} B^{\prime} \leq \operatorname{dim} B_{0} / A \cup C \leq \operatorname{card} B_{0}$.

A set $B_{0} \subseteq B$, which is minimal with the property

$$
b \in B \Rightarrow b \text { is dominated by } C \cup B_{0}
$$

need not be indominated over $C$. Look at the following example.

Examples Here are three examples which shows that the ranks $\mathrm{rk}_{A, C}$ do not behave as one might expect. Let $R_{0}$ be the real closure of $\mathbb{Q}$ in $\mathbb{R}$ and let $\mu$ be some positive infinitesimal. Then we have

(i) $\operatorname{rk}_{R_{0}}\left(R_{0}(\pi+\mu, \pi) / R_{0}\right)=2$. But the set $\{\pi+\mu, \pi\}$ is not an $R_{0}$-dominance basis of $R_{0}(\pi+\mu, \pi)$ over $R_{0}$. In particular $\ll R_{0}$ is different from $\triangleleft R_{0}$.

(ii) $\operatorname{rk}_{R_{0}, \mu}(\{\pi+\mu, \pi\})=1=\operatorname{rk}_{R_{0}}\left(\{\pi+\mu, \pi\} / R_{0}\right)$ and 
$\operatorname{rk}_{R_{0},\{\pi, \pi+\mu\}}(\mu)=0 \neq 1=\operatorname{rk}_{R_{0}}(\mu)$

That is: the symmetry

$$
\operatorname{rk}_{A, D}(B)=\mathrm{rk}_{A} B \Rightarrow \operatorname{rk}_{A, B}(D)=\operatorname{rk}_{A}(D)
$$

does not hold in general.

(iii) If $A$ is a subset of $\mathbb{R}$, and $B$ is an arbitrary set, then $\operatorname{rk}_{A, \mathbb{R}}(B) \leq 1$, since $\mathbb{R}$ is Dedekind complete. Hence, if $p=\operatorname{tp}\left(\mu, \pi / R_{0}\right)$ and $(\alpha, \beta)$ is another realization of $p$, we have

$$
\operatorname{rk}_{R_{0}, \mathbb{R}}(\{\alpha, \beta\}) \leq 1<2=\operatorname{rk}_{R_{0}}(\mu, \pi)
$$

Intuitively speaking this means that $p$ cannot be extended to a type of $\mathbb{R}$ "in an independent way".

The next proposition gives a geometric interpretation of $\mathrm{rk}_{A, C}$.

Definition 1.14 Let $R$ be o-minimal and let $C$ be subset of an elementary extension of $R$. Let $p$ be an n-type over $R$. We say that $p$ is a box type over $C$ if $p$ is uniquely determined as an element of $S_{n}(R \cup C)$ by those formulas from $p$ which define the open boxes $\prod_{i=1}^{n}\left(a_{i}, b_{i}\right), a_{i}, b_{i} \in R$.

If $C \subseteq R$, we just say $p$ is a box type.

So if $p$ is a box type over $C$, then $p$ has a unique extension to $S_{n}(R \cup C)$ and the open $R$-definable boxes containing $p$ imply this extension.

Note that if $\bar{a} \in R^{n}$, then $\{t p(\bar{a} / R)\}$ is a neighborhood of $\operatorname{tp}(\bar{a} / R)$, which does not contain an open box.

Proposition 1.15 Let $R$ be o-minimal and let $C$ be a subset of an elementary extension of $R$. If $p \in S_{n}(R)$, then the following conditions are equivalent:

(i) For some (hence for each) realization $\bar{\alpha}$ of $p$ we have $\operatorname{rk}_{R, C}(\bar{\alpha})=n$.

(ii) $p$ is a box type over $C$.

(iii) If $p_{1}, \ldots, p_{n}$ are the projections of $p$ onto the coordinate axis, then each $p_{i}$ is a cut of $R$ and $p$ is the unique n-type over $R \cup C$ containing each $p_{i}$.

Proof Obviously each of the conditions (i) and (ii) imply $\operatorname{dim} p=n$.

(i) $\Rightarrow$ (ii). By induction on $n$. If $n=1$, then $p$ is omitted in $R\langle C\rangle$, thus (ii) holds. For the induction step, let $\bar{\alpha}$ to be an $n-1$-tuple and let $\beta$ be an element, such that $p$ is realized by $\bar{\alpha}^{\wedge} \beta$ with $\operatorname{rk}_{R, C}(\bar{\alpha} \beta)=n$. By the induction hypothesis, $\operatorname{tp}(\bar{\alpha} / R)$ is a box type over $C$. Let $X$ be an $R \cup C$-definable set which contains $\operatorname{tp}(\bar{\alpha}, \beta / R \cup C)$. Since $\operatorname{dim} \bar{\alpha}, \beta / R \cup C=n$, we can suppose that $X$ is an open cell $(F, G)_{Y}$, where $F, G$ and $Y$ are $R \cup C$-definable. We have $F(\bar{\alpha})<\beta$. As $\operatorname{tp}(\beta / R)$ is omitted in $R\langle C \bar{\alpha}\rangle$, there is some $a_{1} \in R$ with $F(\bar{\alpha}) \leq a_{1}<\beta$. Similar we can find some $a_{2} \in R$ with $\beta<a_{2} \leq G(\bar{\alpha})$. Since $t p(\bar{\alpha} / R)$ is a box type over $C$, there is an open $R$-definable box $Y_{0} \subseteq\left\{\bar{b} \in Y \mid F(\bar{b}) \leq a_{1}, a_{2} \leq G(\bar{b})\right\}$ with $t p(\bar{\alpha} / R) \in Y_{0}$. Finally $Y \times\left(a_{1}, a_{2}\right)$ is an open box, which contains $t p(\bar{\alpha}, \beta / R \cup C)$ and which is contained in $X$. 
(ii) $\Rightarrow$ (i). We do again an induction on $n$. If $n=1$, then (ii) implies that $p$ is omitted in $R\langle C\rangle$, thus $\operatorname{rk}_{R, C}(\alpha)=1$ for all realizations $\alpha$ of $p$. Assume $p \in S_{n}(R)$ is a box type over $C$ and $\bar{\alpha} \beta$ is a realization of $p$. Certainly $t p(\bar{\alpha} / R)$ is a box type over $C$ and by the induction hypothesis $\operatorname{rk}_{R, C}(\bar{\alpha})=n-1$. We have to show that $\operatorname{tp}(\beta / R)$ is omitted in $R\langle C \bar{\alpha}\rangle$ : Let $F$ be an $R \cup C$-definable map, say $F(\bar{\alpha})<\beta$. Let $Y \subseteq R^{n-1}$ be an open box and let $a_{1}<a_{2} \in R$ with $p \in Y \times\left(a_{1}, a_{2}\right)$ and $Y \times\left(a_{1}, a_{2}\right) \subseteq\left\{\left(\bar{b}, b^{\prime}\right) \in R^{n} \mid F(\bar{b})<b^{\prime}\right\}$. That is $F(\bar{b}) \leq a_{1}$ for all $\bar{b} \in Y$, hence $F(\bar{\alpha}) \leq a_{1}<\beta$.

(ii) $\Leftrightarrow$ (iii) If $p_{1}, \ldots, p_{n}$ are the projections of $p$ and each $p_{i}$ is a cut over $R$, then the intersection of all open boxes containing $p$ in $S_{n}(R \cup C)$ is the set of all $n$-types $q \in S_{n}(R \cup C)$ which contain $p_{1}, \ldots, p_{n}$.

The next corollary and the subsequent remark will not be used later on. They relate the notion "box type" to the real spectrum (cf. [1]), for the reader who is aquainted with this point of view. Recall that quantifier elimination for real closed fields says that for every real closed field $R$, the natural map $S_{n}(R) \longrightarrow$ Sper $R[t], t=\left(t_{1}, \ldots, t_{n}\right)$ is a bijection. We say that an element $p \in$ Sper $R[t]$ is a box type if the corresponding $n$-type is a box type.

Corollary 1.16 If $R$ is a real closed field and $p \in \operatorname{Sper} R[t], t=\left(t_{1}, \ldots, t_{n}\right)$ is a box type such that $R$ is archimedean in the real closure of $p$, then $p$ is minimal and maximal in Sper $R[t]$.

Proof Since $\operatorname{dim} p=n, p$ is minimal in Sper $R[t]$. On the other hand, if $q \in$ Sper $R[t]$ is different from $p$ then there is an open box $B$ containing $p$ and not containing $q$. Since $R$ is archimedean in the real closure of $p$, we can find a smaller open box $B^{\prime}$ containing $p$ with $\overline{B^{\prime}} \subseteq B$. Hence $p$ does not specialize to $q$.

Observe that the converse of Corollary 1.16 fails in general. The reason is that a semi-algebraic homeomorphism $R^{n} \longrightarrow R^{n}$ respects the topology of Sper $R[t]$ - hence minimal, maximal points are mapped to minimal, maximal points - but not the property " $p$ is a box type".

In other words, box types cannot be detected with the topology of Sper $R[t]$.

Proposition 1.17 Let $R \prec \mathfrak{M}$ and let $B, C \subseteq \mathfrak{M}$ such that $B$ is $\ll_{R, C}$-independent. Then $\operatorname{tp}(B / R \cup C)$ is the unique extension of $t p(B / R)$. In particular $t p(B / R \cup C)$ is an heir of $t p(B / R)$ (cf. [4], p. 292, for the definition of "heir")

Proof We may assume that $B=\left\{b_{1}, \ldots, b_{n}\right\}$ is finite and we do an induction on $n$. If $n=1$, then $\operatorname{tp}\left(b_{1} / R \cup C\right)$ is the unique extension of $\operatorname{tp}\left(b_{1} / R\right)$, since $b_{1} \rtimes_{R} C$. In the induction step we have: $\operatorname{tp}\left(b_{1}, \ldots, b_{n} / R \cup C\right)$ is the unique extension of $t p\left(b_{1}, \ldots, b_{n} / R\right)$ (from the induction hypothesis) and $t p\left(b_{n+1} / R \cup\right.$ $C \cup\left\{b_{1}, \ldots, b_{n}\right\}$ ) is the unique extension of $t p\left(b_{n+1} / R\right)$ (since $B$ is $\ll_{R, C^{-}}$ independent). These two properties are equivalent to the property that $t p$ $\left(b_{1}, \ldots, b_{n+1} / R \cup C\right)$ is the unique extension of $t p\left(b_{1}, \ldots, b_{n+1} / R\right)$. 
1.2 Behavior of $\ll_{R}$ under base change

First a reminder on the functional version of the Marker-Steinhorn Theorem. Recall that an elementary extension $R \prec S$ of o-minimal structures is called tame, if every $s \in S$, which is $R$-bounded, is infinitely close to an element of $R$ wit respect to $R$.

Theorem 1.18 Let $R \prec S$ be a tame extension of o-minimal expansions of fields. Let $V$ be the convex hull of $R$ in $S$ and let $\lambda: S \longrightarrow R \cup\{\infty\}$ be the place according to $V$. Furthermore let $X \subseteq S^{n}$ and $F: X \longrightarrow S$ be definable in $(S, V)$ with parameters from $S$. For a subset $Y$ of $S^{n}$ let $H(Y):=\bigcup_{y \in Y} y+\mathfrak{m}_{V}^{n}$ denote the set of all points of $S^{n}$ which are infinitely close to a point of $Y$ with respect to $R$. Then

(i) The composed map

$$
\lambda F: F^{-1}(V) \cap R^{n} \longrightarrow S^{n} \stackrel{F}{\longrightarrow} S \stackrel{\lambda}{\longrightarrow} R \cup\{\infty\}
$$

is $R$-definable. $\lambda F$ is the unique map $F^{-1}(V) \cap R^{n} \longrightarrow R$ with the property $(\lambda F)(\bar{a})=\lambda(F(\bar{a}))\left(\bar{a} \in R \cap F^{-1}(V)\right)$.

(ii) There is a decomposition $R^{n}=E \cup D \cup D^{\prime} \cup C$ of $R^{n}$ in R-definable sets, such that:

(a) $F$ is positive infinite on $H(D)$.

(b) $F$ is negative infinite on $H\left(D^{\prime}\right)$.

(c) $F-(\lambda F)_{S}$ is infinitesimal on $H(C)$ and

(d) $\operatorname{dim} E<n$.

Proof This is [5], Theorem 3.3.

Proposition 1.19 Let $R \prec S$ be o-minimal expansions of fields and let $B$ be from an elementary extension of $S$ such that $b 丸_{R} S$ for all $b \in B$.

(i) If $B$ is $\ll s$-independent then $B$ is $R_{R}$-independent.

(ii) If $R$ is tame in $S$, then $B$ is $\ll_{S}$-independent if and only if $B$ is $\ll_{R}$-independent.

Proof (i) Suppose $B$ is $\ll_{S}$-independent and not $\ll_{R}$-independent. By induction on $n$ we may assume that there are $b, b_{1}, \ldots, b_{n}$ such that $\left\{b_{1}, \ldots, b_{n}\right\}$ is $\ll_{R}$-independent and such that $F\left(b_{1}, \ldots, b_{n}\right)$ and $b$ realize the same cut of $R$ for some $R$-definable map $F: R^{n} \longrightarrow R$. By assumption, $F\left(b_{1}, \ldots, b_{n}\right)$ and $b$ realize the same cut of $S$, hence $\left\{b, b_{1}, \ldots, b_{n}\right\}$ is $\ll_{S}$-dependent, a contradiction.

(ii) Now suppose $R$ is tame in $S$. Let $V$ be the convex hull of $R$ in $S$ and let $\lambda: S \longrightarrow R \cup\{\infty\}$ be the place according to $V$. Suppose $B$ is $\ll_{R}$-independent and not $\ll S$-independent. Again, by induction we find $b, b_{1}, \ldots, b_{n} \in B$ such that $\left\{b_{1}, \ldots, b_{n}\right\}$ is $\ll_{S}$-independent, but for some $S$-definable map $F: S^{n} \longrightarrow S$, the element $F\left(b_{1}, \ldots, b_{n}\right)$ induces the same cut over $S$ as $b$. Let $Z:=F^{-1}(V) \cap R^{n}$ 
and $\lambda F: Z \longrightarrow R$ as in Theorem 1.18 (i). Let $R^{n}=E \cup D \sqcup D^{\prime} \cup C$ of $R^{n}$ be a decomposition as in 1.18(ii).

Since $F\left(b_{1}, \ldots, b_{n}\right)=b$ is $R$-bounded, there is an $S$-definable set $Z_{0}$ such that $F$ is $R$-bounded on $Z$ and such that $Z_{0} \in t p\left(b_{1}, \ldots, b_{n}\right)$. By Proposition 1.15 and since $\left\{b_{1}, \ldots, b_{n}\right\}$ is $\ll_{S}$-independent, there is an $S$-definable, open box $O$ such that $\bar{O} \subseteq Z_{0} \backslash E$ and such that $O \in t p\left(b_{1}, \ldots, b_{n}\right)$. Since $b_{i} \rtimes_{R} S$ $(1 \leq i \leq n)$, we may shrink $O$ so that $O$ is $R$-definable. From Theorem 1.18 (ii) we get that $F-(\lambda F)_{S}$ has values in $\mathfrak{m}_{V}$ on $H(\bar{O}) \supseteq \bar{O}_{S}$. But then also $F\left(b_{1}, \ldots, b_{n}\right)-\lambda F\left(b_{1}, \ldots, b_{n}\right)$ is infinitesimal with respect to $R$. Since the cut of $b$ over $R$ is not definable and $F\left(b_{1}, \ldots, b_{n}\right)$ realizes this cut, also $\lambda F\left(b_{1}, \ldots, b_{n}\right)$ realizes this cut. Since $\lambda F$ is $R$-definable, $\left\{b, b_{1}, \ldots, b_{n}\right\}$ is $\ll_{R}$-dependent.

Lemma 1.20 Let $R \prec S$ be o-minimal expansions of fields and let $b$ be from an elementary extension of $S$.

(i) If $S$ is dense in $S\langle b\rangle$ and $b 丸_{R} S$ then $R$ is dense in $R\langle b\rangle$.

(ii) If $R$ is dense in $R\langle b\rangle$ and $S$ does not contain infinitesimal elements with respect to $R$, then $S$ is dense in $S\langle b\rangle$.

Proof (i) Suppose there are $\alpha, \beta \in R\langle b\rangle, \alpha<\beta$ with $(\alpha, \beta) \cap R=\emptyset$. We may assume that $\alpha, \beta \notin R$. Since $S$ is dense in $S\langle b\rangle$ there is some $s \in S$ with $\alpha<s<\beta$, thus $\operatorname{tp}(s / R)=\operatorname{tp}(\alpha / R)$. Since $\alpha \in R\langle b\rangle \backslash R$ there is an $R$-definable map $f: S\langle b\rangle \longrightarrow S\langle b\rangle$ such that $f(\alpha)=b$. Hence $f(s)$ realizes $t p(b / R)$, a contradiction.

(ii) Suppose $S$ is not dense in $S\langle b\rangle$ and $S$ does not contain infinitesimal elements with respect to $R$. Take $\alpha, \beta \in S\langle b\rangle \backslash S$ with $\alpha<\beta$ such that $(\alpha, \beta) \cap S=\emptyset$, in particular $t p(\alpha / S)=t p(\beta / S)$. Let $f: S \longrightarrow S$ be $S$-definable such that $f(\alpha)=b$. Then $f$ is strictly monotonic on the realizations of $t p(\alpha / S)$, hence $\gamma:=f(\beta) \neq b$ is a realization of $\operatorname{tp}(b / S)$. Say $b<\gamma$. Since $S$ does not contain infinitesimal elements with respect to $R$ there is some $m \in R$ such that $0<m<\gamma-b$. Then $b<b+m<\gamma$ and there is no element in $R$ between $b$ and $b+m$.

Proposition 1.21 Let $R \prec S$ be an o-minimal expansions of fields and let $B, D \subseteq$ $S$. Let $B$ be $\ll_{R}$-independent such that $R$ is neither dense nor tame in $R\langle b\rangle$ for all $b \in B$. If $D$ is another $\ll_{R}$-independent set such that $R$ is dense in $R\langle d\rangle$ for each $d \in D$, then $R\langle B\rangle$ is dense in $R\langle B \cup D\rangle$ and $B \cup D$ is $\ll_{R}$-independent.

Proof We may assume that $B=\left\{b_{1}, \ldots, b_{n}\right\}$ and $D=\left\{d_{1}, \ldots, d_{k}\right\}$ are finite. First observe that $R$ is archimedean in $R\langle B\rangle$, otherwise by induction, $b_{n}$ is infinitely close to some $c \in R\left\langle b_{1}, \ldots, b_{n-1}\right\rangle$ and $R$ is archimedean in $R\left\langle b_{1}, \ldots, b_{n-1}\right\rangle$. But then either $b_{n}$ has a definable type over $R$ or $c$ and $b_{n}$ have the same type over $R$, a contradiction to our assumption.

By Lemma 1.20 (ii) applied to $R \prec R\langle B\rangle$ and $d_{1}, R\langle B\rangle$ is dense in $R\left\langle B, d_{1}\right\rangle$. By Lemma 1.20(ii) applied to $R \prec R\left\langle B, d_{1}\right\rangle$ and $d_{2}, R\left\langle B, d_{1}\right\rangle$ is dense in $R\left\langle B, d_{1}, d_{2}\right\rangle$. Continuing in this way we see that $R\langle B\rangle$ is dense in $R\langle B \cup D\rangle$.

Now we prove by induction on $n$ that $B \cup D$ is $\ll_{R}$-independent. Suppose we know that $\left\{b_{1}, \ldots, b_{n-1}\right\} \cup D$ is $\ll_{R}$-independent. Suppose $t p\left(b_{n} / R\right)$ is realized in 
$R\left\langle\left\{b_{1}, \ldots, b_{n-1}\right\} \cup D\right\rangle$. Since $B$ is $\ll_{R}$-independent, $\operatorname{tp}\left(b_{n} / R\left\langle b_{1}, \ldots, b_{n-1}\right\rangle\right)$ is realized in $R\left\langle\left\{b_{1}, \ldots, b_{n-1}\right\} \cup D\right\rangle$. Since $R\left\langle b_{1}, \ldots, b_{n-1}\right\rangle$ is dense in $R\left\langle\left\{b_{1}, \ldots, b_{n-1}\right\} \cup\right.$ $D\rangle$, also $R\left\langle b_{1}, \ldots, b_{n-1}\right\rangle$ is dense in $R\langle B\rangle$. By Lemma 1.20 (i), $R$ is dense in $R\left\langle b_{n}\right\rangle$ a contradiction.

\section{$2 V$-limits}

Let $K \subseteq L$ be ordered fields. In this section we study elements $b$ of $L \backslash K$ which become limits of Cauchy sequences of $K$ after passing to some residue field of a convex valuation ring $V$ of $K$. It turns out that this property only depends on the cut that $b$ generates over $K$, these cuts are then called $V$-limits.

We first recall some notions from [9]. If $X$ is a totally ordered set, then a cut $p$ of $X$ is a tuple $p=\left(p^{L}, p^{R}\right)$ with $X=p^{L} \cup p^{R}$ and $p^{L}<p^{R}$. If $Y \subseteq X$ then $Y^{+}$denotes the cut $p$ of $X$ with $p^{R}=\{x \in X \mid x>Y\}$. $Y^{+}$is called the upper edge of $Y$. Similarly the lower edge $Y^{-}$of $Y$ is defined.

Definition 2.1 Let $p$ be a cut of an ordered abelian group K, The convex subgroup

$$
G(p):=\{a \in K \mid a+p=p\}
$$

of $K$ is called the invariance group of $p\left(\right.$ here $\left.a+p:=\left(a+p^{L}, a+p^{R}\right)\right)$.

If $K$ is an ordered field, then the convex valuation ring

$$
V(p):=\{a \in K \mid a \cdot G(p) \subseteq G(p)\}
$$

is called the invariance valuation ring of $p$. If $s \notin K$ is from an ordered field extension of $K$ then we write $G(s / K)$ and $V(s / K)$ for the invariance group and the invariance ring of the cut induced by s on $K$.

Definition 2.2 Let $K$ be a divisible ordered abelian group and let $p$ be a cut of $K$. We may define the signature of $p$ as

$\operatorname{sign} p:= \begin{cases}1 & \text { if there is a convex subgroup } G \text { of } K \text { and some } a \in K \text { with } p=a+G^{+} \\ -1 & \text { if there is a convex subgroup } G \text { of } K \text { and some } a \in K \text { with } p=a-G^{+} \\ 0 & \text { otherwise }\end{cases}$

Since $K$ is divisible we cannot have $a+G^{+}=b+H^{-}$for $a, b \in K$ and convex subgroups $G, H$ of $K$. Hence the signature is well defined.

In what follows the units of a $\operatorname{ring} A$ will be denoted by $A^{*}$.

Definition 2.3 Let $K$ be an ordered field and let $V \subseteq K$ be a convex valuation ring with maximal ideal $\mathfrak{m}_{V}$. A cut $p$ of $K$ is called $a V$-limit if $\operatorname{sign} p=0$ and if there is some $a \in K^{*}$ such that $G(p)=a \cdot \mathfrak{m}_{V}$. Observe that $V(p)=V$ in this case.

If in addition $G(p)=\mathfrak{m}_{V}$ and $\mathfrak{m}_{V}^{+} \leq p \leq V^{+}$, then $p$ is called a proper $V$-limit. Observe that $\mathfrak{m}_{V}^{+}<p<V^{+}$in this case, as $\operatorname{sign} p=0$. 
An element $b$ from an ordered field extension $L$ of $K$ is called a (proper) $V$-limit if $b \notin K$ and if the cut of $b$ induced on $K$ is a (proper) $V$-limit.

The next proposition states some reformulations of the notion "proper $V$-limit". First some notations. If $K$ is an ordered field, then a sequence $\left(a_{\alpha}\right)_{\alpha<\lambda}$ of elements of $K$ is called a Cauchy sequence, if it is a Cauchy sequence with respect to the order topology of $K$. Observe that for a non-trivial convex valuation ring $V$ of $K$, a Cauchy sequence with respect to $V$ in the valuation theoretic sense (cf. [8]) is a Cauchy sequence in our sense. Recall, if $\left(a_{\alpha}\right)_{\alpha<\lambda}$ is a Cauchy sequence, then a subsequence of $\left(a_{\alpha}\right)_{\alpha<\lambda}$ is a Cauchy sequence with respect to $V$ in the valuation theoretic sense.

An element $b$ from an ordered field extension of $K$ is the limit of a Cauchy sequence $\left(a_{\alpha}\right)_{\alpha<\lambda}$ of $K$ if

$$
\forall \varepsilon \in K, \varepsilon>0 \exists \alpha_{0}<\lambda \forall \alpha>\alpha_{0}\left|b-a_{\alpha}\right|<\varepsilon .
$$

If $T$ is an o-minimal extension of the theory of real closed fields, then a convex valuation ring $V$ of a model $R$ of $T$ is called $T$-convex, if $V$ is the convex hull of an elementary substructure of $R$. In this case, every maximal definably closed subfield $K \subseteq V$ is an elementary substructure of $R$ (cf. [2]).

If $T$ is the theory of real closed fields, then all convex subrings of $R$ are $T$-convex.

Proposition 2.4 Let $L \subseteq M$ be an extension of ordered fields, let $W \subseteq M$ be a convex subring and let $V:=W \cap L$. Let $K \subseteq W$ be a subfield such that $K / \mathfrak{m}_{W}=V / \mathfrak{m}_{W}$. The following are equivalent for every $b \in M$ :

(i) $b$ is a proper $V$-limit.

(ii) $b \in W^{*}$ and $b / \mathfrak{m}_{W}$ is the limit of a Cauchy sequence of $V / \mathfrak{m}_{V}$ without limits in $V / \mathfrak{m}_{V}$.

(iii) $b$ is the limit of a Cauchy sequence of $K$ without limits in $K$.

(iv) $b \notin K$ and $K$ is dense in the ordered group $K+b K$.

(v) $b \notin K, \operatorname{sign}(b / K)=0$ and $G(b / K)=\{0\}$.

Proof We may assume that $b>0$.

(i) $\Rightarrow$ (v) First we prove that $b \notin V+\mathfrak{m}_{W}$. Suppose $b-a \in \mathfrak{m}_{W}$ for some $a \in V$, say $a<b$. Since $\operatorname{sign} b / L=0$ and $G(b / L)=\mathfrak{m}_{V}$, there is some $c \in V, c>\mathfrak{m}_{V}$ with $a+c<b$. Hence $\mathfrak{m}_{V}<c<b-a \in \mathfrak{m}_{W}$ in contradiction to $V=L \cap W$.

This proves $b \notin K$ and for all $c \in K, a \in V$ with $c-a \in \mathfrak{m}_{W}$ we have $c<b$ iff $a<b$.

Let $c \in K, c>0$. We prove that $c \notin G(b / K)$. Let $v \in V$ with $c-v \in \mathfrak{m}_{W}$, say $v<c$. Then $v>\mathfrak{m}_{V}$, since $c>0$. Since $G(b / L)=\mathfrak{m}_{V}$ there is some $a \in V^{>0}$ such that $a<b<a+v$. Let $c_{1} \in K$ with $c_{1}-a \in \mathfrak{m}_{W}$. Then also $a+v-\left(c+c_{1}\right) \in \mathfrak{m}_{W}$ and by what we have shown, $a<b<a+v$ implies $c_{1}<b<c_{1}+c$. Thus $c \notin G(b / K)$ as desired.

It remains to show that $\operatorname{sign}(b / K)=0, \operatorname{say} \operatorname{sign}(b / K) \geq 0$. Since $G(b / K)=0$ it is enough to find for every element $c \in K$ with $c<b$ an element $c_{1} \in K, c_{1}>0$ 
with $c+c_{1}<b$. Let $a \in V$ with $c-a \in \mathfrak{m}_{W}$. Then $a<b$ and from $\operatorname{sign}(b / L)=$ $0, G(b / L)=\mathfrak{m}_{V}$ we get some $v \in V, v>\mathfrak{m}_{V}$ with $a+v<b$. Take $c_{1} \in K$ with $c_{1}-v \in \mathfrak{m}_{W}$. Then $c_{1}>0$ and $c+c_{1}<b$ since $c+c_{1}-(a+v) \in \mathfrak{m}_{W}$.

(v) $\Rightarrow(\mathrm{i})$. First we prove that $b \notin L+\mathfrak{m}_{W}$. Suppose $b-a \in \mathfrak{m}_{W}$ for some $a \in L$. Since $\operatorname{sign}(b / K)=0$, there is some $v \in V$ with $b<v$. But then also $a \in V$. Let $c \in K$ with $a-c \in \mathfrak{m}_{W}$. Then $b-c \in \mathfrak{m}_{W}$ and there is no element in $K$ between $b$ and $c$. This implies that the cut of $b$ over $K$ is definable, a contradiction to $\operatorname{sign} b / K=0$.

Hence $b \notin L+\mathfrak{m}_{W} \supseteq V+\mathfrak{m}_{W}=K+\mathfrak{m}_{W}$. We prove $G(b / L)=\mathfrak{m}_{V}$. First let $v \in \mathfrak{m}_{V}, v \geq 0$ and suppose there is some $l \in L$ with $b<l<b+v$. Then $l-b \in \mathfrak{m}_{W}$ in contradiction to $b \notin L+\mathfrak{m}_{W}$. Hence $\mathfrak{m}_{V} \subseteq G(b / L)$. Conversely let $a \in V, a>\mathfrak{m}_{V}$ and take $c \in K$ with $a-c \in \mathfrak{m}_{W}$. Then $c>0$ and since $G(b / K)=0$ there is some $c_{1} \in K, c_{1}>0$ with $c_{1}<b<c_{1}+c$. Let $a_{1} \in V$ with $a_{1}-c_{1} \in \mathfrak{m}_{W}$. Then $a_{1}<b<a_{1}+a$, hence $a \notin G(b / L)$.

Thus we know $G(b / L)=\mathfrak{m}_{V}$ and it remains to show that $\operatorname{sign}(b / L)=0$. Suppose there is some $a \in V$ such that the cut $\eta$ of $b$ over $L$ is $a \pm \mathfrak{m}_{V}^{+}$, say $\eta=a+\mathfrak{m}_{V}^{+}$. Let $c \in K$ with $a-c \in \mathfrak{m}_{W}$. Then $c<b$. Since $\operatorname{sign}(b / K)=0$ there is some $c_{1} \in K, c_{1}>0$ with $c+c_{1}<b$. Let $a_{1} \in V$ with $c_{1}-a_{1} \in \mathfrak{m}_{W}$. Then $a+a_{1}<b$ and $a_{1}>\mathfrak{m}_{W} \supseteq \mathfrak{m}_{V}$, in contradiction to $\eta=a+\mathfrak{m}_{V}^{+}$.

So we know that (i) is equivalent to (v). The equivalences (i) $\Leftrightarrow$ (ii) $\Leftrightarrow$ (iii) and (iv) $\Leftrightarrow(\mathrm{v})$ are easy and left to the reader.

Remarks Observe that an ordered field $K$ need not be dense in $K(b)$ if $b$ is the limit of a Cauchy sequence of $K$ without limits in $K$. For example if $K=\mathbb{Q}$, $\varepsilon \neq 0$ is infinitesimal and $b=\sqrt{2+\varepsilon}$. Also, a field $K$ as in Proposition 2.4 cannot be found inside $V$ in general. For example if $L=\mathbb{Q}(\sqrt{2}+\varepsilon)$, where $\varepsilon$ is infinitesimal and $V$ is the convex hull of $\mathbb{Q}$ in $L$. Then $\mathbb{Q}$ is the unique subfield of $V$ and $V / \mathfrak{m}_{V} \cong \mathbb{Q}(\sqrt{2})$.

Here is another reformulation of the notion "proper $V$-limit" in terms of so-called distinguished Cauchy sequences as explained in [8], section D:

If $\left(K, V_{0}\right)$ is a valued field, then a sequence $\left(a_{\alpha}\right)_{\alpha<\lambda}$ is called distinguished Cauchy sequence if $\left(a_{\alpha}\right)_{\alpha<\lambda}$ is a pseudo Cauchy sequence of the valued field ( $\left.K, V_{0}\right)$, such that $a_{\alpha} \in V_{0}$ for all $\alpha$ and such that for some valuation ring $V$ of $K$ with $V_{0} \varsubsetneqq V$ the $\left(a_{\alpha}-a_{\beta}\right) / V_{0}^{*}$ are unbounded in the convex subgroup $V^{*} / V_{0}^{*}$ of $K^{*} / V_{0}^{*}$.

We call $V$ the valuation ring associated to $\left(a_{\alpha}\right)_{\alpha<\lambda}$.

Corollary 2.5 In the situation of Proposition 2.4, let $V_{0} \varsubsetneqq V$ be another convex valuation ring. Then $b$ is a proper $V$-limit if and only if $b$ is the pseudo limit of a distinguished pseudo Cauchy sequences of the valued field $\left(L, V_{0}\right)$ which does not have a limit in $L$ and which has $V$ as associated valuation ring.

Proof Easily from Proposition 2.4.

Proposition 2.6 Let $L \subseteq M$ be an extension of ordered fields, let $W \subseteq M$ be a convex subring and let $V:=W \cap L$. Let $K \subseteq W$ be a subfield such that $K / \mathfrak{m}_{W}=V / \mathfrak{m}_{W}$ and let $b \in M$. Then 
(i) $b$ is $a$ V-limit if and only if there are $a_{1}, a \in L, a \neq 0$ such that $a_{1}+a b$ is $a$ proper $V$-limit.

(ii) If $b$ is $a V$-limit and $a V^{\prime}$-limit, where $V^{\prime}$ is a convex valuation ring of $L$, then $V=V^{\prime}$.

(iii) If $L, M$ are models of an o-minimal extension $T$ of the theory of real closed fields, $K, L \prec M$ and if $V$ is $T$-convex, then $K$ is dense in $K\langle b\rangle$ if $b$ is a proper $V$-limit.

Proof (i) If $a_{1}, a \in L, a \neq 0$, then a straightforward computation shows that $G\left(a_{1}+a b / L\right)=a \cdot G(b / L)$ and $\operatorname{sign}(b / L)=\operatorname{sign}\left(a_{1}+a b / L\right)$.

So if $a_{1}, a \in L, a \neq 0$, such that $a_{1}+a b$ is a proper $V$-limit, then $\operatorname{sign}(b / L)=$ $\operatorname{sign}\left(a_{1}+a b / L\right)=0$ and from $G\left(a_{1}+a b / L\right)=\mathfrak{m}_{V}$ we get $G(b / L)=\frac{1}{a} G(a b / L)=$ $\frac{1}{a} G\left(a_{1}+a b / L\right)=\frac{1}{a} \cdot \mathfrak{m}_{V}$, hence $b$ is a $V$-limit.

Conversely if $b$ is a $V$-limit, $a_{0} \in L^{*}$ and $G(b / L)=a_{0} \cdot \mathfrak{m}_{V}$, then $G\left(b \cdot a_{0}^{-1} / L\right)=$ $\mathfrak{m}_{V}$, so there is $a_{1} \in L$ with $a_{1}<b \cdot a_{0}^{-1}<a_{1}+1$. Then $a_{1}-b \cdot a_{0}^{-1}$ is a proper $V$-limit and we may take $a:=-a_{0}^{-1}$.

(ii) If $b$ is a $V$-limit and a $V^{\prime}$-limit, where $V^{\prime}$ is a convex valuation ring of $L$, then $G(b / L)=a \cdot \mathfrak{m}_{V}$ and $G(b / L)=a^{\prime} \cdot \mathfrak{m}_{V^{\prime}}$ for some $a, a^{\prime} \in L^{*}$. But then $\mathfrak{m}_{V^{\prime}}=\frac{a}{a^{\prime}} \mathfrak{m}_{V}$ and this is only possible if $V=V^{\prime}$.

(iii) Suppose $b$ is a proper $V$-limit and $K$ is not dense in $K\langle b\rangle$. Let $a, c \in K\langle b\rangle$ with $a<c$ and $(a, c) \cap K=\emptyset$. We may assume that $a, c \notin K$. There is a $K$-definable map $f: K \longrightarrow K$ such that $f(a)=b$. As $a$ and $c$ realize the same cut over $K, b$ and $f(c)$ realize the same cut over $K$. Moreover $f$ is strictly monotonic in $[a, c] \subseteq K\langle b\rangle$, say $b<f(c)$. Since $\operatorname{sign} b / K=0$ by Proposition $2.4(\mathrm{v})$, there is some $d \in K$ with $0<d<f(c)-b$. As $(b, f(c)) \cap K=\emptyset$ we get $d \in G(b / K)$, a contradiction to $G(b / K)=0$ (cf. Proposition 2.4(v)).

Proposition 2.7 Let $T$ be an o-minimal expansion of fields in the language $\mathscr{L}$, let $R \models T$ and let $\mathscr{V}$ be a set of $T$-convex valuation rings of $R$. For each $V \in \mathscr{V}$ let $K_{V} \subseteq V$ be a maximal definably closed subfield of $V$. Let $S \succ R$ and for each $V \in \mathscr{V}$ let $B_{V} \subseteq S$ be a set of proper $V$-limits.

Then $\bigcup_{V \in \mathscr{V}} B_{V}$ is $\ll_{R}$-independent if and only if $B_{V}$ is $\ll_{K_{V}}$-independent for all $V \in \mathscr{V}$.

Proof We write $B:=\bigcup_{V \in \mathscr{V}} B_{V}$. By Proposition 2.4 each $b \in B_{V}$ has signature 0 over $K_{V}$. Hence $b \Varangle_{K_{V}} R$ for all $b \in B_{V}$. So if $B$ is $\ll_{R}$-independent, then $B_{V}$ is $\ll_{R}$-independent and by Proposition $1.19, B_{V}$ is $\ll_{V}$-independent for all $V \in \mathscr{V}$.

For the converse we may assume that $\mathscr{V}$ is finite, say $\mathscr{V}=\left\{V_{1}, \ldots, V_{n}\right\}$ and $V_{1} \varsubsetneqq \cdots \varsubsetneqq V_{n}$. Let $B_{i} \subseteq B_{V_{i}}$ be finite. It is enough to prove by induction on $n$ that $B=B_{1} \cup \cdots \cup B_{n}$ is $\ll_{R}$-independent if each $B_{i}$ is $\ll_{V_{V}}$-independent. If $n=1$, then we know this from Proposition 1.19(ii).

Induction step. Let $L_{i} \subseteq V_{i}$ be a maximal definably closed subfield of $V_{i}$ with $L_{1} \subseteq \cdots \subseteq L_{n+1}$. From the case $n=1$ we know that each $B_{i}$ is $\ll_{R}$-independent. By what we have above, $B_{i}$ is $\ll_{L_{i}}$-independent. By Proposition 2.4, each $b \in B_{i}$ is a $V_{i} \cap L_{n+1}$-limit. By the induction hypothesis, $B_{1} \cup \cdots \cup B_{n}$ is $\ll L_{n+1}$-independent. 
By Proposition 2.6(iii), $L_{n+1}$ is dense in $L_{n+1}\langle b\rangle$ for each $b \in B_{n+1}$. On the other hand if $b \in B_{1} \cup \cdots \cup B_{n}$, then $L_{n+1}$ is neither dense nor tame in $L_{n+1}\langle b\rangle$. By Proposition 1.21, $B_{1} \cup \cdots \cup B_{n} \cup B_{n+1}$ is $\ll_{L_{n+1}}$-independent. Again by Proposition 1.19, $B_{1} \cup \cdots \cup B_{n} \cup B_{n+1}$ is $\ll_{R}$-independent.

\section{The completion of an o-minimal structure}

Proposition 3.1 Let $T$ be an o-minimal extension of the theory of real closed fields. Let $R \prec \mathcal{M}$ be models of $T$. Then there is a model $S$ of $T$ with $R \prec S \prec \mathcal{M}$, such that:

(i) $R$ is dense in $S$.

(ii) If $R^{\prime}$ is an elementary substructure of $\mathcal{M}, R \prec R^{\prime}$ and if $R$ is dense in $R^{\prime}$, then there is an elementary embedding $R^{\prime} \longrightarrow S$ over $R$.

The embedding in (ii) is unique. If $R \prec S^{\prime} \prec \mathcal{M}$ and $S^{\prime}$ has properties (i) and (ii), then there is a unique $R$-isomorphism $S \longrightarrow S^{\prime}$.

Proof Let $X \subseteq \mathcal{M}$ be the set of all $\alpha \in \mathcal{M}$, such that $R$ is dense in $R\langle\alpha\rangle$. Let $B$ be a $\ll_{R}$-basis of $X$ over $R$. We claim, that $S:=R\langle B\rangle$ has the required properties. Clearly $R$ is an elementary substructure of $S$.

By Proposition 1.21, $R$ is dense in $S$. Let $R^{\prime} \prec \mathcal{M}$ be an elementary extension of $R$, such that $R$ is dense in $R^{\prime}$. Let $B^{\prime}$ be a transcendence basis of $R^{\prime}$ over $R$. Clearly $B^{\prime}$ is an $\ll_{R}$-basis of $R^{\prime}$. By the choice of $B$, the type of every $b^{\prime} \in B^{\prime}$ over $R$ is realized in $R\langle B\rangle$. By Proposition 1.8 we know that $t p\left(B^{\prime} / R\right)$ is realized in $R\langle B\rangle=S$. Hence $t p\left(R^{\prime} / R\right)$ is realized in $S$ and there is an elementary $R$-embedding $R^{\prime} \longrightarrow S$.

Both additions are obvious.

Corollary 3.2 Let $T$ be an o-minimal extension of the theory of real closed fields. Let $R \prec \tilde{R} \prec \mathcal{M}$ be models of $T$, suppose that $R$ is archimedean in $\tilde{R}$ and $\tilde{R}$ is tame in $\mathcal{M}$. We provide $\tilde{R}$ with the topology induced by the ordering of $\tilde{R}$. Let $S$ be the topological closure $\bar{R}$ in this topology. Then $R \prec S \prec \tilde{R} \prec \mathcal{M}$ and $S$ fulfills the conditions (i) and (ii) of Proposition 3.1, both for $R$ and $\tilde{R}$ as well as for $R$ and $\mathcal{M}$. We have

$$
S=\{\alpha \in \tilde{R} \mid R \text { is dense in } R\langle\alpha\rangle\}
$$

Proof Let $R \prec S_{1} \prec \mathcal{M}$ as in Proposition 3.1 and let $S_{1} \prec \tilde{S}_{1} \prec \mathcal{M}$, such that $S_{1}$ is archimedean in $\tilde{S}_{1}$ and $\tilde{S}_{1}$ is tame in $\mathcal{M}$. Since $\tilde{S}_{1}$ and $\tilde{R}$ are isomorphic over $R$ we can suppose that $S_{1} \subseteq \tilde{R}=\tilde{S}_{1}$ ( $T$ is an expansion of $R C F$ ). Since $R$ is archimedean in $\tilde{R}, S_{1}$ is contained in $\bar{R}=S$. If $\alpha \in \bar{R}$, then $R$ is dense in $R\langle\alpha\rangle$. If $\alpha \in \tilde{R}$, such that $R$ is dense in $R\langle\alpha\rangle$, then by Lemma 1.20, the set $S_{1}$ is dense in $S_{1}\langle\alpha\rangle$. By the choice of $S_{1}$ we get therefore $\alpha \in S_{1}$. This proves $S_{1}=S=\{\alpha \in \tilde{R} \mid R$ is dense in $R\langle\alpha\rangle\}$.

Proposition 3.1 applied to a sufficiently large, elementary extension $\mathfrak{M}$ of $R$ yields 
Corollary 3.3 Let $T$ be an o-minimal extension of the theory of real closed fields and let $R$ be a model of $T$. Then there is a model $S \succ R$ with:

(i) $R$ is dense in $S$.

(ii) If $R^{\prime}$ is an elementary extension of $R$ and $R$ is dense in $R^{\prime}$, then there is an elementary embedding $R^{\prime} \longrightarrow S$ over $R$.

The embedding in (ii) is unique. $S$ is uniquely determined up to a unique $R$-isomorphism by conditions (i) and (ii).

The model $S$ in Corollary 3.3 is the largest elementary extension of $R$, such that $R$ is dense in $S$. $S$ is not dense in any proper elementary extension of $S . S$ is called the completion of $R$ and is denoted by $\hat{R}$

We get $S$ by Corollary 3.2 in the following manner: choose $R \prec R_{1} \prec \mathfrak{M}$ such that $R$ is archimedean in $R_{1}$ (i.e. $R_{1}$ is the convex hull of $R$ ), $R_{1}$ is tame in $\mathfrak{M}$ and $\mathfrak{M}$ is $\left|R_{1}\right|^{+}$-saturated. Take

$$
S=\left\{\alpha \in R_{1} \mid R \text { is dense in } R\langle\alpha\rangle\right\}
$$

Since $R$ is dense in $R\langle\alpha\rangle$ if and only if $R$ is dense in the field $R(\alpha)$ (by Propositions 2.4 and 2.6(iii)), the underlying field of the completion of $R$ does not depend on the theory $T$.

If $V$ is a convex valuation ring of $R$ and $\hat{V}$ is the convex hull of $V$ in $\hat{R}$, then the valued field $(\hat{R}, \hat{V})$ is the completion of the valued field $(R, V)$.

\section{Definition of the Pseudo Completion}

Theorem 4.1 Let $T$ be an o-minimal expansion of fields in the language $\mathscr{L}$, let $R \models T$ and let $\mathscr{V}$ be a set of $T$-convex valuation rings of $R$ (the case $R \in \mathscr{V}$ is not excluded). For each $V \in \mathscr{V}$ let $K_{V}, L_{V} \subseteq V$ be maximal definably closed subfields of $V$.

Let $S \succ R$ so that $S$ contains completions $\hat{K}_{V}$ of $K_{V}$ and $\hat{L}_{V}$ of $L_{V}$ for all $V \in \mathscr{V}$. Then

(i) There is an $\mathscr{L}$-isomorphism $\varphi: R\left\langle\bigcup_{V \in \mathscr{V}} \hat{K}_{V}\right\rangle \longrightarrow R\left\langle\bigcup_{V \in \mathscr{V}} \hat{L}_{V}\right\rangle$ over $R$ sending $R\left\langle\hat{K}_{V}\right\rangle$ onto $R\left\langle\hat{L}_{V}\right\rangle$.

(ii) If $K_{V}=L_{V}$ for each $V \in \mathscr{V}$ and $\varphi_{V}$ denotes the unique $\mathscr{L}$-isomorphism $\varphi_{V}: \hat{K}_{V} \longrightarrow \hat{L}_{V}$ over $K_{V}$, then there is a unique $\mathscr{L}$-isomorphism $\varphi:$ $R\left\langle\bigcup_{V \in \mathscr{V}} \hat{K}_{V}\right\rangle \longrightarrow R\left\langle\bigcup_{V \in \mathscr{V}} \hat{L}_{V}\right\rangle$ over $R$ extending all the $\varphi_{V}$.

(iii) The product map

$$
\bigotimes_{V \in \mathscr{V}}\left(R \otimes_{K_{V}} \hat{K}_{V}\right) \longrightarrow S
$$

which sends $\left(\sum r_{1 i} \otimes b_{1 i}\right) \otimes \cdots \otimes\left(\sum r_{k i} \otimes b_{k i}\right)$ to $\left(\sum r_{1 i} \cdot b_{1 i}\right) \cdots \cdots\left(\sum r_{k i} \cdot b_{k i}\right)$ is injective. 
Proof Let $B_{V} \subseteq \hat{K}_{V}$ be a basis of $\hat{K}_{V}$ over $K_{V}$ in the sense of $T$. Since $K_{V}$ is dense in $\hat{K}_{V}, B_{V}$ is $\ll_{V}$-independent. By Proposition 2.7, $B:=\bigcup_{V \in \mathscr{V}} B_{V}$ is $\ll_{R}$-independent. Moreover by Proposition 2.6(iii), for $V \in \mathscr{V}$ and $b \in B_{V}$, the cut of $b$ over $R$ is realized by some $c_{b} \in \hat{L}_{V}$ and in the situation of (ii) we must take $c_{b}:=\varphi_{V}(b)$. Then, by Proposition 1.8 , there is an elementary $R$-embedding $\varphi: R\langle B\rangle \longrightarrow R\left\langle\bigcup_{V \in \mathscr{V}} \hat{L}_{V}\right\rangle$ sending $b$ to $c_{b}$ for each $b \in B_{V}, V \in \mathscr{V}$.

In order to prove that $\varphi$ is surjective and that $\varphi\left(R\left\langle\hat{K}_{V}\right\rangle\right)=R\left\langle\hat{L}_{V}\right\rangle$ it is enough to show that $C_{V}:=\left\{c_{b} \mid b \in B_{V}\right\}$ is a basis of $\hat{L}_{V}$ over $L_{V}$ in the sense of $T$. Clearly $C_{V}$ is independent over $L_{V}$. Let $l \in \hat{L}_{V} \backslash L_{V}, l \notin C_{V}$. Then $l$ is a $V$-limit, so $t p\left(l / K_{V}\right)$ is realized in $\hat{K}_{V}$. Then also $t p(l / R)$ is realized in $R\left\langle B_{V}\right\rangle$, hence $\operatorname{tp}(l / R)$ is realized in $R\left\langle C_{V}\right\rangle$. This means that $C_{V} \cup\{l\}$ is $\ll_{R}$-dependent and by Proposition 1.19, $C_{V} \cup\{l\}$ is $\ll_{L_{V}}$-dependent. Since $l \in \hat{L}_{V}$ and $L_{V}$ is dense in $\hat{L}_{V}$ this is only possible if $l \in L_{V}\left\langle C_{V}\right\rangle$.

This proves (i) and (ii).

(iii). First we show that $R \otimes_{K_{V}} \hat{K}_{V} \longrightarrow S$ is injective, i.e. $R$ and $\hat{K}_{V}$ are linearly disjoint over $K_{V}$. Since $B_{V}$ is $\ll_{K_{V}}$-independent, $B_{V}$ is $\ll_{R}$-independent by Proposition 2.7. Since $t p\left(b / K_{V}\right)$ is omitted in $R$ for all $b \in B_{V}$ it follows that $B_{V}$ is $\ll_{K_{V}, R}$-independent. By Proposition 1.17, $\operatorname{tp}\left(B_{V} / R\right)$ is an heir of $\operatorname{tp}\left(B_{V} / K_{V}\right)$. This property implies that every linear equation with coefficients in $\hat{K}_{V}$ which has a solution in $R$, also has a solution in $K_{V}$. Hence $R$ and $\hat{K}_{V}$ are linearly disjoint over $K_{V}$.

It remains to show that the domains $R \otimes_{K_{V}} \hat{K}_{V}$ are linearly disjoint over $R$. By what we have shown we may identify $R \otimes_{K_{V}} \hat{K}_{V}$ with $R\left[\hat{K}_{V}\right] \subseteq S$. Moreover we may assume that $\mathscr{V}$ is finite, say $\mathscr{V}=\left\{V_{1}, \ldots, V_{n}\right\}$. We write $B_{i}$ for $B_{V_{i}}$. Since $\bigcup_{i=1}^{n} B_{i}$ is $\ll_{R}$-independent, the type $\operatorname{tp}\left(B_{n} / R \cup B_{1} \cup \cdots \cup B_{n-1}\right)$ is an heir over $R$ (c.f. Proposition 1.17). Again it follows that every linear equations with coefficients in $R\left\langle B_{1} \cup \cdots \cup B_{n-1}\right\rangle$ which has a solution in $R\left\langle B_{n}\right\rangle$, also has a solution in $R$. By induction on $n$ we get (iii).

Definition 4.2 In the situation of Theorem 4.1 the model $R\left\langle\bigcup_{V \in \mathscr{V}} \hat{K}_{V}\right\rangle$ of $T$ is called the pseudo completion of $R$ with respect to $\mathscr{V}$.

By Theorem 4.1 this model of $T$ is up to an $R$-isomorphism independent of $S, K_{V}$ and $\hat{K}_{V}$; it can be constructed in the following way. Let $A$ be the $\operatorname{ring}$

$$
A:=\bigotimes_{V \in \mathscr{V}}\left(R \otimes_{K_{V}} \hat{K}_{V}\right) .
$$

Then $A$ is an $R$-algebra without zero divisors and there is an injective $R$-algebra homomorphism $f$ from $A$ into an elementary extension of $R$. Then the pseudo completion is the definable closure of $f(A)$.

If $T$ is the theory of real closed fields then the pseudo completion is the real closure of the quotient field of $A$ with respect to any ordering.

The next proposition describes in what sense the pseudo completion is minimal. 
Proposition 4.3 Let $T$ be an o-minimal expansion of fields in the language $\mathscr{L}$, let $R=T$, let $\mathscr{V}$ be a family of $T$-convex subrings of $R$ and let $R^{\prime}$ be the pseudo completion of $R$ with respect to $\mathscr{V}$. Let $S \succ R$ be an elementary extension of $R$.

(i) Suppose each cut of $R$, which is a $V$-limit for some $V \in \mathscr{V}$ is realized in $S$. Then there is an elementary embedding $R^{\prime} \longrightarrow S$ over $R$.

(ii) For each $V \in \mathscr{V}$, let $W(V)$ be the convex hull of $V$ in $S$. Let $\mathscr{W}$ be a set of $T$-convex valuation rings of $S$ with $W(V) \in \mathscr{W}$ for all $V \in \mathscr{V}$ and let $S^{\prime}$ be the pseudo completion of $S$ with respect to $\mathscr{W}$. Then there is an elementary $R$-embedding $R^{\prime} \longrightarrow S^{\prime}$.

If $\mathscr{W}$ is precisely the set of all $W(V)$ with $V \in \mathscr{V}$ and for each $V \in \mathscr{V}$, the residue field of $V$ is equal to the residue field of $W(V)$, then we can choose this embedding $\varphi$ so that $S^{\prime}$ is the definable closure of $S \cup \varphi\left(R^{\prime}\right)$.

Proof For $V \in \mathscr{V}$ let $K_{V} \subseteq V$ be a maximal definably closed subfield of $V$.

(i). Let $B_{V} \subseteq \hat{K}_{V} \subseteq R^{\prime}$ be a transcendence basis of $\hat{K}_{V}$ over $K_{V}(V \in \mathscr{V})$. Pick some $V \in V$. By assumption and Proposition 2.6(iii), for $b \in B_{V}$ the cut of $b$ over $K_{V}$ is realized in $S$. Since $K_{V}$ is dense in $\hat{K}_{V}, B_{V}$ is $\ll_{K_{V}}$-independent. By Proposition $1.8, \hat{K}_{V}$ can be embedded into $S$ over $K_{V}$. By Theorem 4.1, $R^{\prime}$ can be embedded over $R$ into $S$.

(ii). Let $L_{V} \subseteq W(V)$ be a maximal definably closed subfield of $W(V)$ containing $K_{V}$ for every $V \in \mathscr{V}$. Since $K_{V}$ is archimedean in $L_{V}$ it follows from Lemma 1.20(ii), that there is a (unique) elementary $K_{V}$-embedding $\varphi_{V}$ : $\hat{K}_{V} \longrightarrow \hat{L}_{V}$. By Theorem 4.1 we may assume that $S^{\prime}$ contains the definable closure of $S\left[\bigcup_{V \in \mathscr{V}} \hat{L}_{V}\right]$. By Theorem 4.1, $R^{\prime}$ is $R$-isomorphic to the definable closure of $R\left[\bigcup_{V \in \mathscr{V}} \varphi\left(\hat{K}_{V}\right)\right]$ in $S^{\prime}$.

Now suppose $\mathscr{W}$ is precisely the set of all $W(V)$ with $V \in \mathscr{V}$ and for each $V \in \mathscr{V}$, the residue field of $V$ is equal to the residue field of $W(V)$. Then $L_{V}=K_{V}$ and $S^{\prime}$ is $R$-isomorphic to the definable closure of $S\left[\bigcup_{V \in \mathscr{V}} \hat{K}_{V}\right]$.

By Example 5.11 below, a pseudo completion $R^{\prime}$ of a pure real closed field $R$ is in general not minimal in the sense that any $R$-endomorphism of $R^{\prime}$ is an automorphism. Moreover it is unclear if $R^{\prime}$ is uniquely determined up to an $R$-isomorphism by the minimality demand of Proposition 4.3(i); this is the content of the open problem 5.12 at the end of the paper.

\section{Completion in stages of polynomially bounded structures}

An o-minimal expansion $R$ of a field is called polynomially bounded if every definable function $R \longrightarrow R$ is ultimately bounded by some polynomial. Here all polynomially bounded structures are additionally assumed to have an archimedean prime model. In particular, pure real closed fields are polynomially bounded. If $R$ is polynomially bounded, then every convex subring is Th(R)-convex (cf. [2]). 
Definition 5.1 Let $K$ be an ordered field and let $\mathscr{V}$ be a set of convex valuation rings of $K$. We say that $K$ is complete in stages with respect to $\mathscr{V}$ if all residue fields of elements of $\mathscr{V}$ are complete.

By Proposition 2.4, $K$ is complete in stages with respect to $\mathscr{V}$ if and only if there are no $V$-limits in any ordered field extension of $K$, for all $V \in \mathscr{V}$.

For ordered fields, this definition is more general than the definition of Ribenboim [8]. Let $V$ be a convex valuation ring of an ordered field. Then the valued field $(K, V)$ is complete in stages in the sense of Ribenboim ([8], section D) if and only if $K$ is complete in stages with respect to

$$
\{W \subseteq K \mid W \text { is a convex valuation ring with } V \varsubsetneqq W\}
$$

in our sense. This follows from Corollary 2.5 together with [8], section D, Théorème 3 , which says that the valued field $(K, V)$ is complete in stages if and only if every distinguished pseudo Cauchy sequence of $(K, V)$ has a pseudo limit in $K$.

In this section we construct a completion in stages of $R$ with respect to $\mathscr{V}$ for a polynomially bounded expansion $R$ of a real closed field and a set $\mathscr{V}$ of convex valuation rings of $R$. This is a smallest elementary extension $S$ which is complete in stages with respect to the set of convex hulls of the $V \in \mathscr{V}$. We get $S$ by iterating the construction of the pseudo completion. Before we can do this, we have to compute the residue fields and the value groups of the pseudo completion.

Proposition 5.2 Let $R$ be polynomially bounded and let $s$ be an element from an elementary extension of $R, s \notin R$. The following are equivalent.

(i) $\operatorname{sign}(s / R)=0$.

(ii) If $G$ is a convex subgroup of $(R,+)$, then $G^{+}$is omitted in $R\langle s\rangle$.

(iii) If $W$ is a convex valuation ring of $R\langle s\rangle$, then the value group of $W$ is equal to the value group of $W \cap R$.

Proof Clearly (ii) implies (i). Also (ii) implies (iii), since an element $R\langle s\rangle$ which is not in the value group of $W \cap R$ is the edge of a convex subgroup of $R$.

Conversely suppose $\alpha \in R\langle s\rangle$ realizes $G^{+}$for a convex subgroup $G$ of $(R,+)$. The proposition is proved if we show that $\operatorname{sign}(s / R) \neq 0$ and that $w(\alpha)$ is not in the value group of $V(\alpha / R)$, where $w$ is the valuation of $R\langle s\rangle$ with respect to the convex hull $W$ of $V(\alpha / R)$ in $R\langle s\rangle$.

In order to see this, let $r \in R$ and suppose $\alpha / r \in W^{*}$, say $\alpha / r>0$. Then there are $y, z \in V$ with $0<\alpha / r<y$ and $0<r / \alpha<z$, thus $0<r / z<\alpha<y \cdot r$. Hence $r / z \in G$ and $z y \cdot r / z \notin G$ in contradiction to $z \cdot y \in V(\alpha / R)$.

Hence $w(\alpha)$ is not in the value group of $V(\alpha / R)$. By the valuation property ([3]) there must be some $b \in R$ such that $w(s-b)$ is not in the value group of $V(\alpha / R)$. But then $s-b$ realizes the edge of a convex subgroup of $R$, i.e. $\operatorname{sign}(s / R) \neq 0$. 
Lemma 5.3 Let $R$ be polynomially bounded and let $s$ be from an elementary extension of $R$ with $\operatorname{sign}(s / R)=0$. If $F: R \longrightarrow R$ is $R$-definable with $F(s) \notin R$, then there are $a, b \in R, a<s<b$ such that $F$ is differentiable in $(a, b)$ and for all $r \in R$ with $a<r<b$ we have

$$
G(F(s) / R)=F^{\prime}(r) \cdot G(s / R) .
$$

Proof By $C^{1}$-cell decomposition and since the cut of $s$ over $R$ is not definable, we may assume that $F$ is $C^{1}$ in an open neighborhood of $[a, b]$ for some $a, b \in R$ with $a<s<b$. We write $F^{\prime}$ for the derivative of $F$ in $[a, b]$. If $F$ is a linear map in some interval $(c, d)$ with $c, d \in R, c<s<d$ the lemma holds since $G(y s+z / R)=y G(s / R)$ for all $y, z \in R, y \neq 0$. Hence we may assume that $F^{\prime}(s) \notin R$.

Let $W$ be the convex hull of $V$ in $R\langle s\rangle$. Since $\operatorname{sign}(s / R)=0$, Proposition 5.2 implies that the value group of $W$ is equal to the value group of $V$. Hence there is some $z \in R$ such that $z \cdot F^{\prime}(s) \in W^{*}$. We may replace $F$ by $z \cdot F$, hence we may assume that $F^{\prime}(s) \in W^{*}$, say $F^{\prime}(s)>0$. Since $F^{\prime}(s) \notin R$, Proposition 5.2 gives us $c, d \in V, \mathfrak{m}_{V}<c<d$ with $c<F^{\prime}(s)<d$. By shrinking $(a, b)$ if necessary we may assume that $\left.F\right|_{[a, b]}:[a, b] \longrightarrow[F(a), F(b)]$ is a strictly increasing homeomorphism with $F^{\prime}(x) \in(c, d)$ on $[a, b]$. We prove $G(F(s) / R)=G(s / R)$; this also proves the lemma, since $G(s / R)=F^{\prime}(r) \cdot G(s / R)$ for all $r \in R, a<r<b$.

In order to show $G(s / R) \subseteq G(F(s) / R)$ we take $g \in G(s / R), g>0, r \in R$ with $a<r<s$ and we show that $F(r)+g<F(s)$. Since $F^{\prime}(x)>c$ in $[a, b]$ we know that $F(x)>F(r)+c \cdot(x-r)$ for $x \in(r, b)$. Since $g \in G(s / R)$ and $c \in V^{*}$, we know that $r+g / c<s$, hence $F(x)>F(r)+c \cdot(x-r) \geq F(r)+g$ for $x \in(r+g / c, b)$ and $F(s)>F(r)+g$ as desired.

Conversely let $y \in R$ with $y>G(s / R)$. Then also $y / d>G(s / R)$ and there is some $r \in(a, b)$ with $r<s<r+y / d$. Since $F^{\prime}(x)<d$ in $[a, b]$ we know that $F(x)<F(r)+d \cdot(x-r)$ for $x \in(r, b)$. Hence also $F(x)<F(r)+d \cdot(x-r)<F(r)+y$ for all $x \in R$ with $r<x<\min \{b, r+y / d\}$. Since $r<s<\min \{b, r+y / d\}$ it follows $F(r)<F(s)<F(r)+y$, thus $y \notin G(F(s) / R)$ as desired.

Lemma 5.4 Let $R \prec S$ be polynomially bounded, such that $\operatorname{sign}(s / R)=0$ for all $s \in S \backslash R$. Let $\alpha$ be from an elementary extension of $S$ and let $F: S \longrightarrow S$ be $S$-definable such that $F(\alpha) \notin S$. Suppose $\operatorname{sign}(\alpha / S)=0$ and the cuts of $\alpha$ and $F(\alpha)$ over $R$ are omitted in $S$. Then there is some $c \in R^{*}$ such that $G(F(\alpha) / R)=c \cdot G(\alpha / R)$.

Proof By Lemma 5.3 applied to $S$ and $\alpha$ we get some $s \in S^{*}$ with $G(F(\alpha) / S)=$ $s \cdot G(\alpha / S)$. Let $V=V(\alpha / R)$ and let $W$ be the convex hull of $V$ in $S$. By assumption and by Proposition 5.2, there is some $c \in R^{*}$ such that $c / s \in W^{*}$. Since the cut of $\alpha$ over $R$ is omitted in $S, G(\alpha / S)$ contains $G(\alpha / R)$. Since $S$ does not realize the upper edge of $G(\alpha / R), G(\alpha / S)$ is the convex hull of $G(\alpha / R)$ in $S$. This implies that $V(\alpha / S)$ contains $V=V(\alpha / R)$. Again, since $S$ does not realize the upper edge of $V, V(\alpha / S)$ is the convex hull of $V$ in $S$. Thus $c / s$ is a unit in $V(\alpha / S)=W$ and $s \cdot G(\alpha / S)=s \cdot(c / s) \cdot G(\alpha / S)=c \cdot G(\alpha / S)$. 
By Proposition 5.2, $\operatorname{sign} F(\alpha) / S=0$, hence also $G(F(\alpha) / S)$ is the convex hull of $G(F(\alpha) / R)$ in $S$. Thus $G(F(\alpha) / S)=c \cdot G(\alpha / S)$ implies $G(F(\alpha) / R)=$ $c \cdot G(\alpha / R)$.

Corollary 5.5 Let $R$ be polynomially bounded, let $s_{1}, \ldots, s_{n}$ be from an elementary extension of $R$ with $\operatorname{rk}_{R}\left(s_{1}, \ldots, s_{n}\right)=n$ and $\operatorname{sign}\left(s_{i} / R\right)=0$ for all $i \in\{1, \ldots, n\}$. If $F: R^{n} \longrightarrow R$ is $R$-definable and $F\left(s_{1}, \ldots, s_{n}\right) \notin R$, then $\operatorname{sign}\left(F\left(s_{1}, \ldots, s_{n}\right) / R\right)=0$ and $G\left(F\left(s_{1}, \ldots, s_{n}\right) / R\right)=c \cdot G\left(s_{i} / R\right)$ for some $i \in$ $\{1, \ldots, n\}$ and some $c \in R$.

Proof For $i \in\{1, \ldots, n\}$ the cut of $s_{i}$ over $R$ is omitted in $R\left\langle s_{1}, \ldots, s_{i-1}\right\rangle$. As $\operatorname{sign}\left(s_{i} / R\right)=0$ it follows that $\operatorname{sign}\left(s_{i} / R\left\langle s_{1}, \ldots, s_{i-1}\right\rangle\right)=0$. Then by induction on $n$, Proposition 5.2 implies that every $s \in R\left\langle s_{1}, \ldots, s_{i}\right\rangle \backslash R$ has signature 0 .

Let $\alpha:=F\left(s_{1}, \ldots, s_{n}\right)$. Since $\alpha \notin R$ there is some $i \in\{1, \ldots, n\}$ such that $\alpha \cup$ $\left(\left\{s_{1}, \ldots, s_{n}\right\} \backslash\left\{s_{i}\right\}\right)$ is a $\ll_{R}$-basis of $R\left\langle s_{1}, \ldots, s_{n}\right\rangle$. Say $i=1$. Let $S:=R\left\langle s_{2}, \ldots, s_{n}\right\rangle$. Since the cuts of $\alpha$ and $s_{1}$ over $R$ are omitted in $S$ and $\operatorname{sign}\left(s_{1} / S\right)=0$, we can apply Lemma 5.4. Hence $G(\alpha / R)=c \cdot G\left(s_{1} / R\right)$ for some $c \in R^{*}$.

Now we compute the residue fields and the value groups of convex valuation rings of the pseudo completion of a polynomially bounded structure:

Theorem 5.6 Let $R$ be polynomially bounded and let $S$ be the pseudo completion of $R$ with respect to a set $\mathscr{V}$ of convex subrings of $R$.

(i) Every $s \in S \backslash R$ is a $V$-limit for a unique convex valuation ring $V$ of $R$ and this ring is in $\mathscr{V}$.

(ii) Let $V_{0}$ be any convex valuation ring of $R$. Then the convex hull $W_{0}$ of $V_{0}$ in $S$ is the unique convex valuation ring of $S$ lying over $V_{0}$. The value group of $W_{0}$ is the value group of $V_{0}$ and

(a) if $V_{0} \varsubsetneqq V$ for all $V \in \mathscr{V}$, then the extension $\left(R, V_{0}\right) \subseteq\left(S, W_{0}\right)$ of valued fields is immediate;

(b) if $V \subseteq V_{0}$ for some $V \in \mathscr{V}$, then $W_{0} / \mathfrak{m}_{W_{0}}$ is the pseudo completion of $V_{0} / \mathfrak{m}_{V_{0}}$ with respect to $\left\{V / \mathfrak{m}_{V_{0}} \mid V \in \mathscr{V}, V \subseteq V_{0}\right\}$.

Proof (i) follows from Corollary 5.5, since $S$ is the definable closure of a $\ll_{R^{-}}$ independent set of elements, each being a $V$-limit for some $V \in \mathscr{V}$ (cf. Propositions 2.7 and 2.4). The uniqueness statement holds by Proposition 2.6(ii).

(ii) By (i), every $s \in S \backslash R$ is a $V$-limit for some $V \in \mathscr{V}$, in particular $\operatorname{sign}(s / R)=$ 0 . By Proposition 5.2, no edges of convex subgroups of $R$ are realized in $S$. Consequently $W_{0}$ is the unique convex valuation ring of $S$, lying over $V_{0}$ and $W_{0}$ must have the same value group as $V_{0}$.

In order to see (a) and (b) let $K_{V} \subseteq V$ be a maximal definably closed subfield for each $V \in \mathscr{V} \cup\left\{V_{0}\right\}$.

(a) Suppose $V_{0} \varsubsetneqq V$ for all $V \in \mathscr{V}$. Let $s \in W_{0}$. We have to show that the cut $p$ of $s$ over $K_{V_{0}}$ is definable. Suppose $p$ is not definable. If $G\left(s / K_{V_{0}}\right)=0$, then $s$ is a $V_{0}$-limit by Proposition 2.4. By (i), $s$ is a $V$-limit for some $V \in \mathscr{V}$. Since $V \neq V_{0}$ this is impossible (cf. Proposition 2.6(i)). Hence $G\left(s / K_{V_{0}}\right) \neq 0$ and $V\left(s / K_{V_{0}}\right)$ is a proper convex valuation ring of $K_{V_{0}}$. Since $p$ is omitted in $R, G(s / R)$ is the 
largest convex subgroup of $R$ with $G(s / R) \cap K_{V_{0}}=G\left(s / K_{V_{0}}\right)$. This implies that $V(s / R)$ is a convex valuation ring, lying over $V\left(s / K_{V_{0}}\right)$. As $V\left(s / K_{V_{0}}\right)$ is proper it follows $V(s / R) \subseteq V_{0}$. On the other hand - by (i) $-V(s / R) \in \mathscr{V}$ and this contradicts our assumption on $V_{0}$.

(b) By Theorem 4.1 we may assume that $K_{V_{0}} \subseteq K_{V}$ for every $V \in \mathscr{V}$ with $V_{0} \subseteq V$. For $V \in \mathscr{V}$ with $V \subseteq V_{0}$, any maximal definably closed subfield of $V \cap K_{V_{0}}$ is also a maximal definably closed subfield of $V$ (this is so, since such a field $L$ is archimedean in $V \cap K_{V_{0}}$ and tame in $K_{V_{0}}$ - as $V \cap K_{V_{0}}$ is archimedean in $V$ and $K_{V_{0}}$ is tame in $R$, also $L$ is archimedean in $V$ and tame in $R$ ). So by Theorem 4.1 we may assume that $K_{V} \subseteq K_{V_{0}}$ for all $V \in \mathscr{V}$ with $V \subseteq V_{0}$, too. Let $\mathscr{V}^{\prime}:=\left\{V \in \mathscr{V} \mid V \subseteq V_{0}\right\}$ and let $R^{\prime}:=R\left\langle\bigcup_{V \in \mathscr{V}^{\prime}} \hat{K}_{V}\right\rangle$ be the pseudo completion of $R$ with respect to $\mathscr{V}^{\prime}$.

First we prove (ii) (b) for $\mathscr{V}^{\prime}$ and $W_{0}^{\prime}:=W_{0} \cap R^{\prime}$. By Theorem 4.1 it is enough to show that $K_{V_{0}}\left\langle\bigcup_{V \in \mathscr{V}}, \hat{K}_{V}\right\rangle$ is a maximal definably closed subfield of $W_{0}^{\prime}$. In order to prove this it suffices to take $V_{1}, \ldots, V_{n} \in \mathscr{V}^{\prime}$ and finite subsets $B_{i} \subseteq K_{V_{i}}$ independent over $K_{V_{i}}(1 \leq i \leq n)$ and to show that $K_{V_{0}}\langle B\rangle$ is a maximal definably closed subfield of $W_{0} \cap R\langle B\rangle$. By Proposition 2.7, the $B_{i}$ are mutually disjoint and their union $B$ is $\ll K_{V_{0}}$-independent. Hence for each $b \in B$ the cut $p$ of $b$ over $K_{V_{0}}$ is omitted in $K_{V_{0}}\langle B \backslash\{b\}\rangle$. Since $p$ is not definable, the unique extension to $K_{V_{0}}\langle B \backslash\{b\}\rangle$ is not definable as well. This shows that $K_{V_{0}}\langle B \backslash\{b\}\rangle$ is archimedean in $K_{V_{0}}\langle B\rangle$. Hence, by induction, $K_{V_{0}}$ is archimedean in $K_{V_{0}}\langle B\rangle$ and $K_{V_{0}}\langle B\rangle$ is a subfield of $W_{0}$. Since $R\langle B\rangle$ is generated by $B$ and $\operatorname{dim} R\langle B\rangle / R$ is greater or equal to the dimension of the residue field of $W_{0} \cap R\langle B\rangle$ over $V_{0} / \mathfrak{m}_{V_{0}}$ (cf. [2]), $K_{V_{0}}\langle B\rangle$ must be a maximal definably closed subfield of $W_{0} \cap R\langle B\rangle$.

Hence we know that the residue field of $W_{0}^{\prime}=W_{0} \cap R^{\prime}$ is the pseudo completion of $V_{0} / \mathfrak{m}_{V_{0}}$ with respect to $\left\{V / \mathfrak{m}_{V_{0}} \mid V \in \mathscr{V}, V \subseteq V_{0}\right\}$ and it remains to show that $\left(R^{\prime}, W_{0}^{\prime}\right) \subseteq\left(S, W_{0}\right)$ is immediate. But this follows from a., since by Theorem 4.1, $S$ is the pseudo completion of $R^{\prime}$ with respect to the set of convex hulls of all $V \in \mathscr{V}$ with $V_{0} \varsubsetneqq V$.

Example 5.7 The pseudo completion $R^{\prime}$ of a real closed field $R$ with respect to a set $\mathscr{V}$ of convex valuation rings of $R$, containing $R$, is not complete in general. In particular, if $\mathscr{V}^{\prime}$ denotes the set of convex hulls of elements from $\mathscr{V}$ in $R^{\prime}$, then $R^{\prime}$ need not be complete in stages with respect to $\mathscr{V}^{\prime}$.

To see an example, let $K$ be a real closed field with completion $\hat{K} \neq K$ and let $\Gamma$ be a divisible subgroup of $(\mathbb{R},+)$ containing $1 \in \mathbb{R}$. We also assume that $\Gamma$ is an ordered subgroup of $(K,+)$. In this situation we can equip the generalized power series field $\hat{K}\left(\left(t^{\Gamma}\right)\right)$ with the derivative

$$
\left(\sum a_{\gamma} t^{\gamma}\right)^{\prime}=\sum a_{\gamma} \cdot \gamma \cdot t^{\gamma-1}
$$

Let $R$ be the real closure of $K\left(t^{\gamma} \mid \gamma \in \Gamma\right)$ in $\hat{K}\left(\left(t^{\Gamma}\right)\right)$ and let $V$ be the convex hull of $K$ in $R$. The completion $\hat{R}$ of $R$ is 


$$
\hat{R}=\left\{\sum_{n=0}^{\infty} a_{n} t^{\gamma_{n}} \mid a_{n} \in K, \gamma_{n} \in \Gamma \text { and } \gamma_{n} \rightarrow \infty(n \rightarrow \infty)\right\} .
$$

Hence the pseudo completion of $R$ with respect to $\{V, R\}$ is $\hat{R}\langle\hat{K}\rangle$. We claim that for $x \in \hat{K} \backslash K$, the element

$$
\exp (x \cdot t):=\sum_{i=0}^{\infty} \frac{x^{i}}{i !} t^{i}
$$

is not in $\hat{R}\langle\hat{K}\rangle$. Since $\exp (x \cdot t)$ is in the completion of $\hat{K}\left(t^{\gamma} \mid \gamma \in \Gamma\right) \subseteq \hat{R}\langle\hat{K}\rangle$, this will show the incompleteness of $\hat{R}\langle\hat{K}\rangle$. We use a differential algebraic argument:

Lemma 5.8 Let $K \subseteq L$ be ordinary differential fields of characteristic 0, let $y, x \in L, y \neq 0$ such that $x$ is transcendental over $K$. Suppose $g, h \in K[x]$, with $y^{\prime}=g \cdot y$ and $x^{\prime}=h$. If $g \notin K$ and $\operatorname{deg} g \geq \operatorname{deg} h$, then $y$ and $x$ are algebraically independent over $K$. Here the degree is the degree with respect to $x$.

Proof Suppose $y$ is algebraic over $K(x)$. Let $f_{d-1}, \ldots, f_{0} \in K(x)$ be rational functions, such that

$$
\mu(T):=T^{d}+f_{d-1} T^{d-1}+\cdots+f_{0}
$$

is the minimal polynomial of $y$ over $K(x)$. Then

$$
\begin{aligned}
0=\mu(y)^{\prime} & =d \cdot y^{d-1} \cdot y^{\prime}+f_{d-1}^{\prime} y^{d-1}+f_{d-1} \cdot(d-1) \cdot y^{d-2} y^{\prime}+\cdots+f_{1}^{\prime} y+f_{1} y^{\prime}+f_{0}^{\prime} \\
& =d \cdot g \cdot y^{d}+\left(f_{d-1}^{\prime}+f_{d-1} \cdot(d-1) \cdot g\right) \cdot y^{d-1}+\cdots+\left(f_{1}^{\prime}+f_{1} g\right) y+f_{0}^{\prime}=: \eta(y) .
\end{aligned}
$$

Since $x^{\prime} \in K(x), K(x)$ is a differential subfield of $L$ and $\eta(y)=0$ is an algebraic relation of $y$ over $K(x)$ of degree $d$. Hence $\eta(y)=d \cdot g \cdot \mu(y)$ and a comparison of the constant coefficients with respect to y implies $f_{0}^{\prime}=d \cdot g \cdot f_{0}$. Let $P, Q \in K[T]$ with $f_{0}=P(x) / Q(x), Q(x) \neq 0$. Since $y \neq 0, P(x) \neq 0$. From $f_{0}^{\prime}=d \cdot g \cdot f_{0}$ we get

$$
Q(x) \cdot P(x)^{\prime}-P(x) \cdot Q(x)^{\prime}=d \cdot g \cdot P(x) \cdot Q(x) .
$$

Since $x^{\prime}=h$ and $\operatorname{deg} g \geq \max \{1, \operatorname{deg} h\}, P(x)^{\prime}$ is a polynomial in $x$ of degree $<\operatorname{deg} P+\operatorname{deg} g$. Also $\operatorname{deg} Q(x)^{\prime}<\operatorname{deg} Q+\operatorname{deg} g$, hence $\operatorname{deg}\left(Q(x) \cdot P(x)^{\prime}-P(x)\right.$. $\left.Q(x)^{\prime}\right)<\operatorname{deg} P+\operatorname{deg} Q+\operatorname{deg} g=\operatorname{deg}(d \cdot g \cdot P(x) \cdot Q(x))$, a contradiction.

Now we prove $\exp (x \cdot t) \notin \hat{R}\langle\hat{K}\rangle$. Let $B \subseteq \hat{K}$ be a transcendence basis of $\hat{K}$ over $K$ containing $x$ and let $C$ be a transcendence basis of $\hat{R}$ over $R$. By Proposition 2.7, $B \cup C$ is a transcendence basis of $\hat{R}\langle\hat{K}\rangle$ over $R$ and $B \cap C=\emptyset$. Let $L:=\hat{R}\langle B \backslash\{x\}\rangle$. The field $\hat{R}$ is a differential subfield of $\hat{K}\left(\left(t^{\Gamma}\right)\right)$, equipped with the derivative introduced above (thus $\left(t^{\gamma}\right)^{\prime}=\gamma \cdot t^{\gamma-1}$ for $\gamma \in \Gamma$ ). Since $L$ is obtained from $\hat{R}$ by adjoining constants to $\hat{R}$ and then taking the real closure, 
$L$ is also a differential subfield of $\hat{K}\left(\left(t^{\Gamma}\right)\right)$. Moreover $x$ is transcendental over $L$. Since $\exp (x \cdot t)^{\prime}=x \cdot \exp (x \cdot t)$ and $x^{\prime}=0$, Lemma 5.8 implies that $\exp (x \cdot t)$ and $x$ are algebraically independent over $L$. Hence $\exp (x \cdot t) \notin L\langle x\rangle=\hat{R}\langle\hat{K}\rangle$ as desired.

In the example above, the pseudo completion of $S:=\hat{R}\langle\hat{K}\rangle$ with respect to $\{S, W\}$, where $W$ is the convex hull of $V$, is the completion of $\hat{K}\left(t^{\gamma} \mid \gamma \in \Gamma\right)$.

More generally, if $R$ is polynomially bounded, $\mathscr{V}$ is a set of convex valuation rings of $R$, let $R^{\prime}$ be the pseudo completion of $R$ with respect to $\mathscr{V}$ and let $\mathscr{V}^{\prime}$ be the set of convex hulls of elements from $\mathscr{V}$ in $R^{\prime}$. We write $(R, \mathscr{V})^{\prime}$ for $\left(R^{\prime}, \mathscr{V}^{\prime}\right)$. We define for each ordinal $\alpha$ the pair $\left(R^{(\alpha)}, \mathscr{V}^{(\alpha)}\right)$ by $\left(R^{(0)}, \mathscr{V}^{(0)}\right):=$ $(R, \mathscr{V}),\left(R^{(\alpha+1)}, \mathscr{V}^{(\alpha+1)}\right):=\left(R^{(\alpha)}, \mathscr{V}^{(\alpha)}\right)^{\prime}$ and for a limit ordinal $\alpha$ we take $R^{(\alpha)}=$ $\bigcup_{\beta<\alpha} R^{(\beta)}$ and $\mathscr{V}^{(\alpha)}:=\left\{\bigcup_{\beta<\alpha} V^{(\beta)} \mid V \in \mathscr{V}\right\}$.

Let $V_{0} \subseteq \bigcap_{V \in \mathscr{V}} V$ be a convex subring and let $V_{0}^{(\alpha)}$ be the convex hull of $V_{0}$ in $R^{(\alpha)}$.

Claim The extension $\left(R^{(1)}, V_{0}^{(1)}\right) \subseteq\left(R^{(\alpha)}, V_{0}^{(\alpha)}\right)$ is immediate for all $\alpha \geq 1$.

Proof By induction on $\alpha$, where the limit step is obvious. Suppose we know that $\left(R^{(1)}, V_{0}^{(1)}\right) \subseteq\left(R^{(\alpha)}, V_{0}^{(\alpha)}\right)$ is immediate for some $\alpha \geq 1$. We show that $\left(R^{(\alpha)}, V_{0}^{(\alpha)}\right) \subseteq\left(R^{(\alpha+1)}, V_{0}^{(\alpha+1)}\right)$ is immediate. If $V_{0} \notin \mathscr{V}$, then $V_{0}^{(\alpha)} \notin \mathscr{V}(\alpha)$ and we can apply Theorem 5.6(ii)(a).

Hence we may assume that $V_{0}$ is the least element in $V$. Then also $V_{0}^{(\alpha)}$ is the least element of $\mathscr{V}^{(\alpha)}$. By Theorem 5.6(ii)(b), $V_{0}^{(1)}$ has a complete residue field. By induction, $V_{0}^{(\alpha)}$ has a complete residue field, too. Hence $R^{(\alpha+1)}$ is the pseudo completion of $R^{(\alpha+1)}$ with respect to $\mathscr{V}^{(\alpha)} \backslash\left\{V_{0}^{(\alpha)}\right\}$. But then again by Theorem 5.6(ii)(a),$\left(R^{(\alpha)}, V_{0}^{(\alpha)}\right) \subseteq\left(R^{(\alpha+1)}, V_{0}^{(\alpha+1)}\right)$ is immediate.

From the claim it follows that $R^{(\alpha)}$ can be embedded as a field into the maximal immediate extension of the valued field $\left(R^{(1)}, V_{0}^{(1)}\right)$. Consequently there must be some ordinal $\alpha$ with $R^{(\alpha)}=R^{(\alpha+1)}$.

Definition 5.9 The completion in stages of $R$ with respect to $\mathscr{V}$ is defined to be the elementary extension $R^{(\alpha)}$ for an ordinal $\alpha$ with $R^{(\alpha)}=R^{(\alpha+1)}$.

By construction, the completion in stages is complete in stages with respect to the family of convex hulls of the rings from $\mathscr{V}$. Moreover the properties of the pseudo completion from Theorem 5.6 are inherited by the completion in stages:

Theorem 5.10 Let $R$ be polynomially bounded and let $S$ be the completion in stages of $R$ with respect to a set $\mathscr{V}$ of convex subrings of $R$.

(i) Every $s \in S \backslash R$ is a $V$-limit for a unique convex valuation ring $V$ of $R$ and this ring is in $\mathscr{V}$.

(ii) Let $V_{0}$ be any convex valuation ring of $R$. Then the convex hull $W_{0}$ of $V_{0}$ in $S$ is the unique convex valuation ring of $S$ lying over $V_{0}$. The value group of $W_{0}$ is the value group of $V_{0}$ and 
(a) if $V_{0} \varsubsetneqq V$ for all $V \in \mathscr{V}$, then the extension $\left(R, V_{0}\right) \subseteq\left(S, W_{0}\right)$ of valued fields is immediate;

(b) if $V \subseteq V_{0}$ for some $V \in \mathscr{V}$, then $W_{0} / \mathfrak{m}_{W_{0}}$ is the completion in stages of $V_{0} / \mathfrak{m}_{V_{0}}$ with respect to $\left\{V / \mathfrak{m}_{V_{0}} \mid V \in \mathscr{V}, V \subseteq V_{0}\right\}$.

(iii) Let $S^{\prime}$ be an elementary extension of $R$ and for each $V \in \mathscr{V}$ let $W_{V}^{\prime}$ be the convex hull of $V$ in $S^{\prime}$. If $S^{\prime}$ is complete in stages with respect to $\left\{W_{V}^{\prime} \mid V \in \mathscr{V}\right\}$, then there is an elementary embedding $\varphi: S \longrightarrow S^{\prime}$ over $R$.

Proof For an ordinal $\alpha$, let $R^{(\alpha)}$ and $\mathscr{V}^{(\alpha)}$ be as in the construction of $S$ above. First we prove (ii). Let $V_{0}$ be any convex valuation ring of $R$ and let $V_{0}^{(\alpha)}$ be the convex hull of $V_{0}$ in $R^{(\alpha)}$. By induction on $\alpha$ we get from Theorem 5.6 that $V_{0}^{(\alpha)}$ is the unique convex valuation ring of $R^{(\alpha)}$, lying over $V_{0}$ and the value group of $V_{0}^{(\alpha)}$ is the value group of $V_{0}$. Moreover item (ii)(a) follows immediately from Theorem 5.6(ii)(a) by induction on $\alpha$.

(ii)(b) By Theorem 5.6(ii)(b) for every ordinal $\alpha, V_{0}^{(\alpha+1)} / \mathfrak{m}_{V_{0}^{(\alpha+1)}}$ is the pseudo completion of $V_{0}^{(\alpha)} / \mathfrak{m}_{V_{0}^{(\alpha)}}$ with respect to $\left\{V^{(\alpha)} / \mathfrak{m}_{V_{0}^{(\alpha)}} \mid V \in \mathscr{V}, V \subseteq V_{0}\right\}$. By induction on $\alpha$ we get that $V_{0}^{(\alpha)} / \mathfrak{m}_{V_{0}^{(\alpha)}}$ is the $\alpha$-fold iterated pseudo completion of $V_{0} / \mathfrak{m}_{V_{0}}$ with respect to $\left\{V / \mathfrak{m}_{V_{0}} \mid V \in \mathscr{V}, V \subseteq V_{0}\right\}$. This easily implies (ii)(b)

(i) The uniqueness statement is obviously true.

By induction on $\alpha$ we prove that every $x \in R^{(\alpha)} \backslash R$ is a $V$-limit for some $V \in \mathscr{V}$. For $\alpha=1$ we know this from Theorem 5.6(i). For limit ordinals there is nothing to do. Now suppose $x \in R^{(\alpha+1)}$. If the cut of $x$ over $R$ is realized in $R^{(\alpha)}$, then by the induction hypothesis, $x$ is a $V$-limit for some $V \in \mathscr{V}$. Hence we may assume that the cut of $x$ over $R$ is omitted in $R^{(\alpha)}$. Since $R^{(\alpha+1)}$ is the pseudo completion of $R^{(\alpha)}$ with respect to $\mathscr{V}^{(\alpha)}$, Theorem 5.6(i) gives us some $V \in \mathscr{V}$ such that $x$ is a $V^{(\alpha)}$-limit. Thus $\operatorname{sign}\left(x / R^{(\alpha)}\right)=0$ and for some $a \in R^{(\alpha)}$, $G\left(x / R^{(\alpha)}\right)=a \cdot \mathfrak{m}_{V^{(\alpha)}}$. Since the cut of $x$ over $R$ is omitted in $R^{(\alpha)}$, we have $\operatorname{sign}(x / R)=0$.

Since the value group of $V^{(\alpha)}$ is the value group of $V$, there is some $r \in R$ such that $r / a \in\left(V^{(\alpha)}\right)^{*}$. Hence $a \cdot \mathfrak{m}_{V^{(\alpha)}}=r \cdot \mathfrak{m}_{V^{(\alpha)}}$. Since the cut of $x$ over $R$ is omitted in $R^{(\alpha)}$ and $G(x / R)^{+}$is omitted in $R^{(\alpha)}, G\left(x / R^{(\alpha)}\right)=r \cdot \mathfrak{m}_{V^{(\alpha)}}$ is the convex hull of $G(x / R)$. Since $\mathfrak{m}_{V^{(\alpha)}}$ is the convex hull of $\mathfrak{m}_{V}$, it follows that $G(x / R)=r \cdot \mathfrak{m}_{V}$. Together with $\operatorname{sign}(x / R)=0$, this means that $x$ is a $V$-limit.

(iii) Since $W_{V}^{\prime}$ is the convex hull of $V$ and the residue field of $W_{V}^{\prime}$ is complete, for every maximal definably closed subfield $K$ of $V$ there is a completion of $K$ inside $W_{V}^{\prime}$. By Theorem 4.1, there is an elementary embedding of $R^{(1)}$ into $S^{\prime}$ over $R$. By an obvious induction this can be iterated until we reach the completion in stages.

If $\mathscr{V}$ is finite of size $n$, then $R^{(n)}$ is complete in stages with respect to $\mathscr{V}^{(n)}$. This follows from Theorem 5.6 by induction on $n$ : if $\mathscr{V}=\left\{V_{1}, \ldots, V_{n}\right\}$ with $V_{1} \varsubsetneqq \cdots \varsubsetneqq V_{n}$, then by Theorem 5.6(ii) (b), $V_{1}^{(1)}$ has a complete residue field. Thus $R^{(2)}$ is the pseudo completion of $R^{(1)}$ with respect to $\left\{V_{2}^{(1)}, \ldots, V_{n}^{(1)}\right\}$. 
Moreover $V_{1}^{(1)} \subseteq V_{1}^{(2)}$ is immediate by Theorem 5.6(ii)(a) Hence by induction, $R^{(n)}$ is complete in stages with respect to $\mathscr{V}^{(n)}$.

Example 5.11 One might ask if the pseudo completion or the completion in stages $S$ of a real closed field $R$ with respect to a set of convex valuation rings is minimal in the sense that every $R$-embedding $S \longrightarrow S$ is surjective. This is not true in general. Look at the following example.

Let $R=R_{0}\langle\mu\rangle$ be the real closure of $\mathbb{Q}(\mu)$, where $\mu$ is infinitesimal and let $S$ be the pseudo completion of $R$ with respect to the valuation $\operatorname{ring} V:=$ the convex hull of $\mathbb{Q}$ in $R$. Then $S$ is $\mathbb{R}\langle\mu\rangle$, which is the completion in stages of $R$ with respect to the valuation ring $V$, too. We now construct a proper real closed subfield $R$ of $\mathbb{R}\langle\mu\rangle$, which contains $\mu$ and which is isomorphic over $R_{0}\langle\mu\rangle$ to $S$. In particular $R$ realizes every cut of $R_{0}$.

Let $T \subseteq \mathbb{R}$ be a transcendence basis over $R_{0}$ and let $B=\left\{b_{1}, b_{2}, \ldots\right\}$ be a countable subset of $T$. Let

$$
R:=R_{0}\left\langle(T \backslash B) \cup\left\{\mu, b_{1}+\mu b_{2}, b_{2}+\mu b_{3}, \ldots\right\}\right\rangle .
$$

Then $b_{1} \notin R$, otherwise there is some $n \in \mathbb{N}$ such that $b_{1} \in R_{1}:=R_{0}\langle(T \backslash$ $\left.B) \cup\left\{\mu, b_{1}+\mu b_{2}, \ldots, b_{n}+\mu b_{n+1}\right\}\right\rangle$. But then $b_{1}, \ldots, b_{n+1}, \mu \in R_{1}$, hence $R_{1}$ has transcendence degree $\geq n+2$ over $R_{0}\langle T \backslash B\rangle$, which is not possible.

$R$ is isomorphic to $S$ over $R_{0}\langle(T \backslash B) \cup\{\mu\}\rangle$, the isomorphism is given by sending $b_{i}$ to $b_{i}+\mu \cdot b_{i+1}$ (observe that $T \cup\{\mu\}$ is $\ll_{R_{0}}$-independent and $b_{i}$ and $b_{i}+\mu b_{i+1}$ realize the same cut over $R_{0}$. Then use Proposition 1.8).

Open Problem 5.12 Let $S$ be a real closed field containing $\mathbb{R}$, of transcendence degree 1 over $\mathbb{R}$. Let $S_{0}$ be a real closed subfield of $S$ which realizes every cut of $\mathbb{Q}$. Is $S_{0}$ isomorphic to $S$ ?

More general, let $S$ be the pseudo completion of a real closed field and let $\varphi: S \longrightarrow S$ be an $R$-algebra homomorphism. Let $S_{0}$ be a real closed field with $\varphi(S) \subseteq S_{0} \subseteq S$. Is $S_{0}$ isomorphic to $S$ over $R$ ? In the example above, $R$ is the real closure of $\mathbb{Q}(\mu)$, where $\mu$ is infinitesimal and $S$ is the pseudo completion of $R$ with respect to the valuation ring $V:=$ the convex hull of $\mathbb{Q}$ in $R$. Then $S=\mathbb{R}\langle\mu\rangle$ also is the completion in stages of $R$ with respect to $\{V\}$.

\section{References}

1. Bochnak, J., Coste, M., Roy, M.F.: Real Algebraic Geometry. Ergebnisse der Mathematik und ihrer Grenzgebiete, vol. 36. Springer, Berlin Heidelberg New York (1998)

2. van den Dries, L., Lewenberg, A.H.: T-convexity and tame extension. J. Symb. Logic 60(1), 74-101 (1995)

3. van den Dries, L., Speissegger, P.: The field of reals with multisummable series and the exponential function. Proc. Lond. Math. Soc. 81(3), 513-565 (2000)

4. Hodges, W.: Model Theory. Encyclopedia of mathematics and its applications, vol. 42. Cambridge university Press, Cambridge (1993)

5. Marker, D., Steinhorn, C.: Definable types in $o$-minimal theories. J. Symb. Logic 59, 185-198 (1994) 
6. Pillay, A., Steinhorn, C.: Definable sets in ordered structures I. Trans. Am. Math. Soc. 295, 565-592 (1986)

7. Prieß-Crampe, S.: Angeordnete Strukturen: Gruppen, Körper, projektive Ebenen. Ergebnisse der Mathematik und ihrer Grenzgebiete vol. 98. Springer, Berlin Heidelberg New York (1983)

8. Ribenboim, P.: Théorie des valuations. Les Presses de l'Université de Montréal, Montreal (1964)

9. Tressl, M.: Model Completeness of o-minimal Structures expanded by Dedekind Cuts. J. Symb. Logic 70(1), 29-60 (2005)

10. van der Waerden, B.L.: Algebra I. Springer, Berlin Heidelberg New York (1966) 